\title{
On-Machine Optical Probe Based on Discrete Rotational Symmetric Triangulation
}

\author{
Xu Zhang $\mathbb{D}^{1},{ }^{1}$ Jianxiang Wang $\mathbb{D},{ }^{1}$ Kai Fei $\mathbb{D}^{2},{ }^{2}$ and Quan Zhang $\mathbb{D}^{1}$ \\ ${ }^{1}$ Shanghai University, School of Mechatronic Engineering and Automation, Department of Mechanical Engineering and Automation, \\ No. 149, Yanchang Road, Shanghai 200072, China \\ ${ }^{2}$ United Automotive Electronic Systems Co., Ltd., No. 555, Rongqiao Road, Shanghai 201206, China
}

Correspondence should be addressed to Xu Zhang; xuzhang@shu.edu.cn and Quan Zhang; lincolnquan@shu.edu.cn

Received 16 May 2018; Accepted 28 June 2018; Published 19 July 2018

Academic Editor: Juan C. Jauregui-Correa

Copyright (C) 2018 Xu Zhang et al. This is an open access article distributed under the Creative Commons Attribution License, which permits unrestricted use, distribution, and reproduction in any medium, provided the original work is properly cited.

\begin{abstract}
In the field of modern manufacturing, probes have been playing a more and more crucial role. However, contact probes are unfit for measuring objects with soft surfaces or very tiny cavities and require radius compensation of stylus. In comparison, noncontact probes such as optical probes do not have to consider these problems and are well-performed in measurement. However, existing optical probes (triangulation) may fail to detect objects with rich spatial structures (due to the occlusion effect) and highly reflective curved surfaces (due to existence of highlight spots). Considering the problems, an optical probe based on discrete rotational symmetric triangulation is proposed in this paper. The emitting laser beam of the probe is the rotational axis and the five receiving optical paths (lenses and mirrors) are rotationally symmetrically distributed around the laser axis and are coimaged on a CMOS camera. Results of simulations and experiments show that the new-established prototype is designed with good robustness under various conditions such as different surface characteristics and different inclination degrees. The probe enables conducting real-time on-machine measurement without directional dependency with a resolution of $39 \mu \mathrm{m}$ and repeatability accuracy of $0.72 \mu \mathrm{m}$.
\end{abstract}

\section{Introduction}

With the rapid development of modern manufacturing, complex parts have been more and more widely adopted in ship, automobile, aerospace, and other fields. Dimensional size and surface morphology of parts such as turbine blades, horological gears, and tiny biomedical device require being measured by probes. Because probes play a crucial role in modern industry, a number of related researches have been contributed in this area and significant probes for various purposes have been proposed accordingly. They can be divided into contact probes and noncontact probes.

Existing contact probes (mechanical probes, touchtrigger probes, scanning probes, etc.) own quite mature mechanical structures and electronic systems with high accuracy and reliability. Mechanical probes are commonly used in miniaturized measuring machines with low precision, which are easy to operate. Wim P. van Vliet et al. proposed a mechanical probe system for a coordinate measuring machine $(\mathrm{CMM})$. The proposed prototype can achieve $1 \mu \mathrm{m}$ in the measurement uncertainty when the speed is $70 \mathrm{~mm} / \mathrm{s}$. It shows great advantage in accuracy and speed compared with congeneric mechanical probes [1]. Except for mechanical probes, touch-trigger probes are also popular, because of their fast measuring speed and good flexibility. When approaching the surface of the workpiece, the stylus of the touch-trigger probe is skewed and the trigger signal is transmitted to the infrared receiver or the radio receiver. The transmitted electrical signal of the either type is ultimately transmitted to the corresponding controller. Wen-long Li et al. conducted inspection on impeller blades using an on-machine touchtrigger probe, OMP40. The measuring profile errors of both suction surface and pressure surface of the impeller blades are within less than $0.14 \mathrm{~mm}$ [2]. Del Guerra et al. presented a touch-trigger probe model for CNC lathes, which is based on a simple electrical contact. The tests of the probe have shown a repeatability of $3 \mu \mathrm{m}$ [3]. Takaaki Oiwa et al. proposed a miniaturized touch-trigger probe system with one illuminating fiber and three bundles of receiving fibers in the 
stylus stem. Their experiment results show that the measuring resolution is less than $0.5 \mu \mathrm{m}$ and the measurement force is less than $0.9 \mathrm{mN}$ in all directions [4]. A serial of touchtrigger probes such as TP20, TP 200, and TP7M EP was launched by Renishaw, a leading probe manufacturer for precision measurement. Compared with kinematic touchtrigger probes such as TP20, both TP200 and TP 7M EP are electronic probes using strain gauge technology, which have higher accuracy and do not need mechanical makeand-break of contact point [5]. Italian corporation Marposs launched high performance MIDA TT-serials touch-trigger probes, such as TT25, TT60, and TT25h, primarily used for 3D measurements on milling machines, sharpeners, and grinding machines [6]. The third popular touch probes are scanning probes, which can also collect continuous points for profile inspection with good accuracy and repeatability besides discrete triggered point. Rui-Jun Li et al. proposed a long-stroke contact scanning probe for micro/nano-CMM, which is based on an elastic mechanism and two high precision sensors. The probe can achieve a uniform stiffness of $0.5 \mathrm{mN} / \mathrm{um}$ and a standard deviation of $30 \mathrm{~nm}$ [7].

Contact probes including mechanical probes, touchtrigger probes, and scanning probes mentioned above have four common shortcomings. First, contact probes are unfit for the measurement of soft and fragile surfaces as they touch objects for data collection. They are required to remain below certain limitations arising from the elastic and plastic deformation at the contact point [8]. Second, contact probes have to consider another problem in terms of the size of the measured microcavity, because it is difficult for a touchtrigger probe to measure tiny holes when it is smaller than the probe tip. Third, approach-direction-dependent errors of touch-trigger probes cannot be neglected and usually exceed those from other sources [9]. For the purpose of reducing the errors, the direction of approach is required to remain as normal as possible to the surface. Finally, radius compensation for contact probes is required since the obtained data are the coordinates of the probe center instead of those of the contact point. Therefore, technical difficulties are attached to probe design. Related researches in terms of radius compensation are involved in a number of literature [10-13].

Compared with contact probes, noncontact probes do not have mechanical contact with measured surfaces in the process of measuring, which possesses high scanning speed and is fit for measuring of fragile surfaces and small cavities. Marco Silvestri et al. used laser scanners in machine tools to implement free-form parts machining and quality control. The system with the probes can achieve a resolution of less than $2 \mu m$ and repeatability less than $18 \mu m$ [14]. Xicong Zou et al. integrated a chromatic confocal measurement probe with an ultraprecision diamond turning machine to conduct on-machine measurement. Experiments show that the probe owns a resolution of $83.33 \mathrm{~nm}$ [15]. However, confocal microscopes run up against the problem that the amplitude of the signal under the best-focus condition changes when the intensity of the returning light changes, which results in measuring errors [16]. White light interferometry is faster than confocal microscopes as it only requires a scan in a single (vertical) direction. However, it does not work well especially while measuring step with height less than the coherence length of the light source, which is caused by the batwing effect $[17,18]$. In addition, scanning white light interferometry also does not give correct surface height even though the step height is significantly greater than the coherence length, primarily resulting from a phase change caused by optical field diffracting around the discontinuity. The measuring error is thereby relatively significant [18]. Optical triangulation is a well-performed method without requirement for the size of the measured objects.

Laser triangulation [19-23] is one of the most common methods for commercial 3D measurement. It is found that laser triangulation measurement applied for on-machine measurement has advantages of simple structure, fast measuring speed, large measuring range, flexibility, and strong real-time processing ability. Soichi Ibaraki et al. used an optical triangulation-based scanning probe to conduct five-axis on-machine measurement. The laser displacement sensor achieved the standard uncertainty of sphere center position of about $0.5 \mu \mathrm{m}$ [24]. Haibo Zhang et al. proposed a typical symmetric rotational triangulation sensor based on an object space mirror, which simplifies structure and reduces costs, but its big size is the main drawback that keeps it from mounting on a machine [22]. Michael Demeyere et al. proposed a method for robust noncontact diameter determination of spherical objects based on laser triangulation. Experiment results showed that accuracy is about $1 \%$ for spherical objects of 10-30 cm in diameter [25]. However, traditional triangulation probes suffer from a serious drawback of occlusion; namely, shadings exist in a scene due to spatial structure of objects, which is commonly seen on objects with step surfaces, free-form surfaces, cavities, etc. [26]. To overcome the problem, single laser triangulation system requires additional mechanical degree of freedom (DOF) to ensure the proper angle between probe and the workpiece surface. On the basis of the single triangulation, dual-laser triangulation system optimizes itself by imaging from two directions. Edmond Wai Yan So et al. successively proposed an online duallaser triangulation system and its improved version for fast building models in $[27,28]$. A similar method proposed by Lijiang Zeng et al. used an acoustooptic deflector (AOD) as a scanner to allow a diffracted beam to scan an object from two directions and the shadow effects can also be mitigated [29]. A dual-laser system does to some degree mitigate the occlusion problem compared with single laser triangulation system, while it still does not completely solve it. Another serious problem for traditional triangulation emerges in the case of measuring specular surfaces, which are generally seen in the process of detecting objects such as automobile body, precision bearing ball, and optical component. The problem is required to be carefully considered, as reflection of surroundings on highly reflective curved surfaces has great impact on the measurement, since specular objects distort the appearance of other objects nearby and create an indirect view on the original objects. The problem gives rise to the existence of unknown highlight spots in sight, which will lead to the failure of imaging. What is more, 


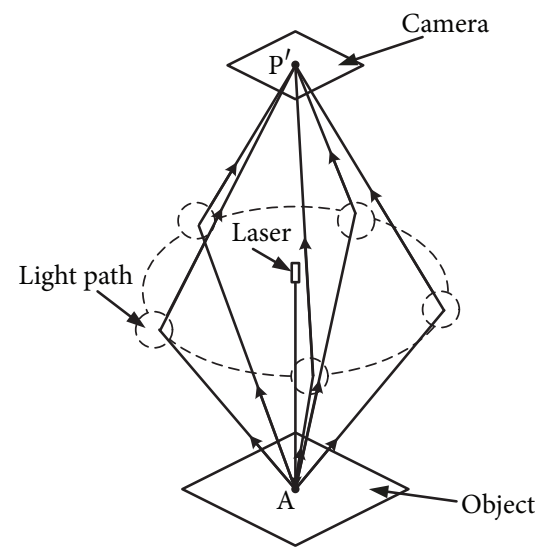

(a)

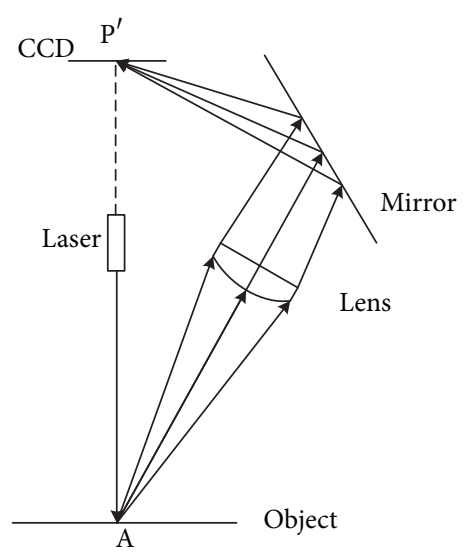

(b)

FIGURE 1: Schematic diagram of the discrete rotational triangulation. (a) Overall light path model. (b) Single light path model.

almost all the desired laser energy is reflected from the direction normal to the surface and therefore is hard to be detected by the sensor in a certain direction $[30,31]$. Therefore, it can be concluded that directional dependence of traditional triangulation is a primary factor that affects measuring performance in the case of detecting objects with highly reflective curved surfaces or with complicated spatial structure.

To overcome the direction-dependent problem of laser triangulation measurement, the traditional method is to increase the degree of freedom (DOF) of the manipulator loaded by the measuring device, but additional mechanical design and cost are required. Another way is to change the equipment structure from the standpoint of the flexibility of the measuring device. In this paper, an optical probe based on discrete rotational symmetric triangulation is proposed. The emitting laser beam of the probe is the rotational axis and the five receiving optical paths (lenses and mirrors) are rotationally symmetrically distributed around the laser axis and are coimaged on a CMOS camera. The system can effectively solve the direction-dependent problem existing in traditional triangulation because the optical probe with discrete rotational symmetric characteristics can detect at least one light spot in all circumstances. The rotational symmetric optics can measure some surfaces with rich spatial high-frequency components and important feature information such as slopes, holes, and steps.

This paper is organized as follows. In Section 2, the fundamental principle of the optical probe is explained, which is called the discrete rotational symmetric triangulation in this paper. Mathematical model of the probe is then constructed and the imaging principle is shown in this section. Afterwards, simulations under different surface characteristics are conducted with Zemax and preliminary analysis of the results is described in Section 3. In Section 4, the corresponding experiments are conducted, where the method of detecting laser spots are described and the calibration and the depth measurement are conducted.

\section{Discrete Rotational Symmetric Triangulation}

2.1. Principles of the Discrete Rotational Symmetric Triangulation. The principle of the discrete rotational symmetric triangulation is shown in Figure 1(a). The laser beam hits on the surface of the object, and the scattered lights are received by five optical paths and imaged on a common receiving camera. The five received optical paths distribute around the rotation axis in space evenly, with feature of rotational symmetry. The emitted laser beam is the rotational symmetry axis. Each optical path is composed of a lens and a corresponding mirror. The image plane of the receiving camera is perpendicular to the rotational symmetry axis.

The discrete rotational symmetric triangulation conforms to the triangulation measuring principle, as shown in Figure 1(b). The scattered lights from the object point $\mathrm{A}$ are reflected by the mirror and imaged on the sensor. When object point A moves up and down in depth direction, the image points move correspondingly on the image plane. Compared with traditional laser triangulation, the discrete rotational symmetric triangulation changes the optical paths with mirrors so that the optical paths can commonly share a receiving camera and laser. Besides, the rotational symmetry feature makes it possible to measure depth using panoramic ability, which avoids the directional dependence of the traditional triangulation system. Because light path conforms to Scheimpflug tradition, the object can be clearly imaged on the receiving camera with the change of its depth.

2.2. Mathematical Model of the Optics. Triangulation measurement is a measuring method based on the lens imaging process and conducted with obtained ideal imaging results. The ideal imaging requires to meet the need that when the measured object moves, the image points and object points should be always consistent with the imaging formula. The pin-hole imaging is one of the usual imaging model in which image plane, object plane, and principal plane of the lens are parallel to each other. In the imaging model of the 


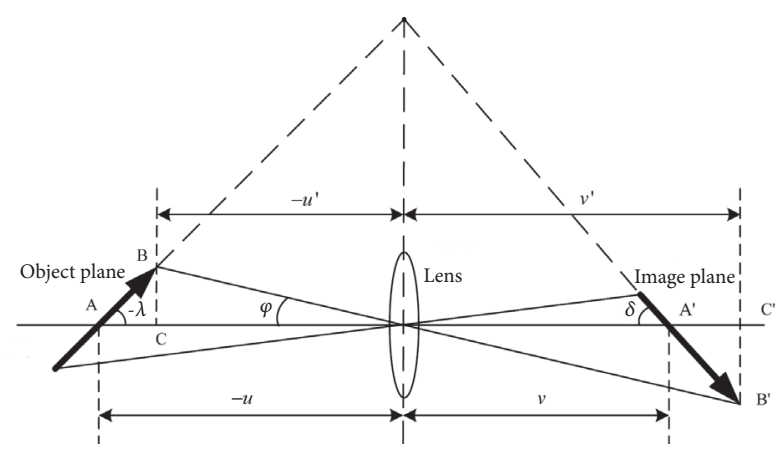

FIGURE 2: Optical path conforming to Scheimpflug condition.

Scheimpflug condition, it can still clearly imaged even when the image plane, the object plane, and the lens are not parallel to each other and intersect in a straight line. The optical path of meridional plane satisfying the Scheimpflug condition is shown in Figure 2.

According to the Gauss law in geometric optics, the relation between $u$ and $v$ is presented as

$$
\frac{1}{v}-\frac{1}{u}=\frac{1}{f}
$$

where $f$ is the focal length of the lens.

As can be seen from Figure 2, $-u$ can be calculated as

$$
-u=-u^{\prime}+B C \cdot \cot (-\lambda)
$$

where $B C=-u^{\prime} \cdot \tan \varphi$; substitute it into (2); $u^{\prime}$ can be calculated as

$$
u^{\prime}=\frac{u}{1+\tan \varphi \cot \lambda}
$$

Similarly, $v^{\prime}$ can be obtained as

$$
v^{\prime}=\frac{v}{1-\tan \varphi \cot \delta}
$$

According to geometric optical knowledge, $u^{\prime}$ and $v^{\prime}$ also conform to Gauss law

$$
\frac{1}{v^{\prime}}-\frac{1}{u^{\prime}}=\frac{1}{f}
$$

Combining the equations above, the following relation can be obtained:

$$
\tan \lambda=\frac{v}{u} \tan \delta
$$

If light paths of triangulation system conform to (6), light spots on measured object can be clearly imaged within limits. The light path of the measurement system in the paper is designed on the basis of the condition.

According to Scheimpflug condition, the following three planes, the plane on which object points are located, the lens principal plane, and the image plane, are required to

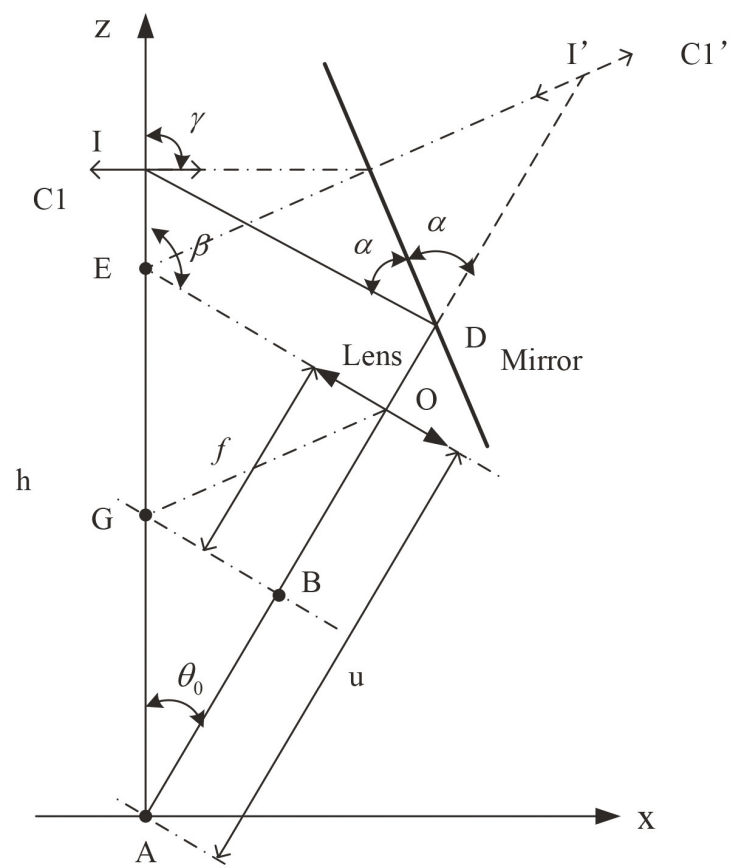

Figure 3: Parametric design of the optical path.

intersect with each other in a line. The parametric model of the measurement system is shown in Figure 3. The object point on the principal optical axis is denoted as A. When scattered laser beams are imaged on the camera $\mathrm{C} 1^{\prime}$ through lens, the actual imaging spot is located at I of the camera $\mathrm{C} 1$ due to the effect of the mirror. The angle between optical axis $\mathrm{AO}$ and $\mathrm{Z}$-axis is $\theta_{0}$. The lens is placed perpendicular to the optical axis $\mathrm{AO}$ at a distance of $u$ from point $\mathrm{A}$, and the angle between it and $\mathrm{Z}$-axis is $\beta$. The extension cord of the lens intersects with $\mathrm{Z}$-axis at point $\mathrm{E}$. The focal length of the lens is denoted as $f$, point B locates at the position of one-time focal length. The line perpendicular to optical axis AO and passing through point B intersects with Z-axis at point G. According to Scheimpflug condition, point $\mathrm{G}$ is located on the line both parallel to the lens and one-time focal length away from the lens. It is also located on the line both passing through the lens center and parallel to the ideal image plane; that is, the OG is the ideal image plane at the position of one-time focal length away from the lens. Consequently, it can be deduced that the line both passing through point $\mathrm{E}$ and parallel to line OG is the ideal image plane of the lens at the current position.

Let the coordinate of object point $\mathrm{A}$ be $(0,0)$; the direction of the lens is $\beta=\pi / 2+\theta_{0}$, the position of point $\mathrm{O}$ is $\left(u \sin \left(\theta_{0}\right), u \cos \left(\theta_{0}\right)\right)$, and that of point $\mathrm{B}$ is $((u-f)$. $\left.\sin \left(\theta_{0}\right),(u-f) \cdot \cos \left(\theta_{0}\right)\right)$; point $\mathrm{G}$ is the intersection point of the line the laser beam located on and the line both parallel to the lens and one-time focal length away from the lens, satisfying the following equation:

$$
\tan (\beta)=\frac{G_{x}-B_{x}}{G_{z}-B_{z}}
$$


where $G_{x}=0$; the coordinate of point $\mathrm{G}$ can be obtained as

$$
\begin{aligned}
G & =\left[\begin{array}{c}
0 \\
(u-f) \cos \left(\theta_{0}\right)-\frac{(u-f) \sin \left(\theta_{0}\right)}{\tan (\beta)}
\end{array}\right] \\
& =\left[\begin{array}{c}
0 \\
\frac{u-f}{\cos \left(\theta_{0}\right)}
\end{array}\right]
\end{aligned}
$$

The direction of $\operatorname{dir} \overrightarrow{G O}$ is

$$
\operatorname{dir} \overrightarrow{G O}=\arctan \left(\frac{G_{x}-O_{x}}{G_{z}-O_{z}}\right)=\arctan \left(\frac{-O_{x}}{G_{z}-O_{z}}\right)
$$

Point E is located on both line EO and Z-axis, satisfying the equation

$$
\tan (\beta)=\frac{E_{x}-O_{x}}{E_{z}-O_{z}}
$$

where $E_{x}=0$; the coordinate of point $\mathrm{E}$ can be obtained as

$$
E=\left[\begin{array}{c}
0 \\
u \cos \left(\theta_{0}\right)-\frac{u \sin \left(\theta_{0}\right)}{\tan (\beta)}
\end{array}\right]=\left[\begin{array}{c}
0 \\
\frac{u}{\cos \left(\theta_{0}\right)}
\end{array}\right]
$$

$\mathrm{I}^{\prime}$ is the intersection point of the principal optical axis and the camera $\mathrm{Cl}^{\prime}$, satisfying the following equation:

$$
\begin{gathered}
\tan \left(\theta_{0}\right)=\frac{I_{x}^{\prime}-A_{x}}{I_{z}^{\prime}-A_{z}}=\frac{I_{x}^{\prime}}{I_{z}^{\prime}} \\
\tan (\operatorname{dir} \overrightarrow{G O})=\tan \left(\operatorname{dir} \overrightarrow{E I^{\prime}}\right)=\frac{E_{x}-I_{x}^{\prime}}{E_{z}-I_{z}^{\prime}}=\frac{-I_{x}^{\prime}}{E_{z}-I_{z}^{\prime}}
\end{gathered}
$$

Solve the set of equations (12) and (13); the coordinate of the point $\mathrm{I}^{\prime}$ can be obtained as

$$
I^{\prime}=\left[\begin{array}{c}
\tan \left(\theta_{0}\right) I_{y}^{\prime} \\
\frac{E_{y} \tan (\operatorname{dir} \overrightarrow{G O})}{\tan (\operatorname{dir} \overrightarrow{G O})-\tan \left(\theta_{0}\right)}
\end{array}\right]
$$

Camera $\mathrm{Cl}^{\prime}$ is mirrored to Camera $\mathrm{C} 1$. It is required that camera $\mathrm{Cl}$ is parallel to $\mathrm{X}$-axis and image point $\mathrm{I}$ is located on $\mathrm{Y}$-axis. DI and $\mathrm{DI}^{\prime}$ are symmetric with each other in terms of the mirror and the angle between them and the mirror is $\alpha$. The direction of ID $\operatorname{dir} \overrightarrow{\mathrm{ID}}$ is

$$
\operatorname{dir} \overrightarrow{\mathrm{ID}}=\pi-2 \alpha+\theta_{0}
$$

Camera $\mathrm{C} 1$ is set perpendicular to $\mathrm{Y}$-axis; that is, $\gamma=\pi / 2$. According to angular symmetry, the equation can be obtained as

$$
\operatorname{dir} \overrightarrow{\mathrm{ID}}-\gamma=\operatorname{dir} \overrightarrow{\mathrm{EI}^{\prime}}-\theta_{0}=\operatorname{dir} \overrightarrow{\mathrm{GO}}-\theta_{0}
$$

The mirror's angle $\alpha$ is

$$
\alpha=\frac{\pi}{4}-\left(\frac{\operatorname{dir} \overrightarrow{\mathrm{GO}}-2 \theta_{0}}{2}\right)
$$

Point $\mathrm{D}$ is the intersection point of the mirror and the principal optical axis, whose position will affect whether point I on camera $\mathrm{Cl}$ can overlap Z-axis or not. $\overrightarrow{\mathrm{ID}}$ is symmetric to $\overrightarrow{\mathrm{DI}^{\prime}}$ in terms of the mirror; thus the length of $\overrightarrow{\mathrm{ID}}$ and $\overrightarrow{\mathrm{DI}^{\prime}}$ is equal. The equation can be obtained as

$$
\begin{aligned}
D_{x} & =|\overrightarrow{\mathrm{ID}}| \cdot \sin (\pi-\operatorname{dir} \overrightarrow{\mathrm{ID}})=|\overrightarrow{\mathrm{ID}}| \cdot \sin \left(2 \alpha-\theta_{0}\right) \\
I_{x}^{\prime} & =D_{x}+\left|\overrightarrow{\mathrm{DI}^{\prime}}\right| \cdot \sin \theta_{0} \\
|\overrightarrow{\mathrm{ID}}| & =\left|\overrightarrow{\mathrm{DI}^{\prime}}\right|
\end{aligned}
$$

Solving the simultaneous equations (18), (19), and (20), we can obtain

$$
D_{x}=I_{x}^{\prime} \cdot \frac{\sin \left(2 \alpha-\theta_{0}\right)}{\sin \left(2 \alpha-\theta_{0}\right)+\sin \theta_{0}}
$$

Point $\mathrm{D}$ is located on the line $\mathrm{AO}$, sufficing for the equation $D_{y}=D_{x} \cdot \cot \theta_{0}$. The coordinate of point $\mathrm{D}$ is

$$
D=\left[\begin{array}{c}
I_{x}^{\prime} \cdot \frac{\sin \left(2 \alpha-\theta_{0}\right)}{\sin \left(2 \alpha-\theta_{0}\right)+\sin \theta_{0}} \\
D_{x} \cdot \cot \theta_{0}
\end{array}\right]
$$

Camera $\mathrm{Cl}$ is on $\mathrm{Z}$-axis; the distance between the camera and $\mathrm{X}$-axis is

$$
h=|\overrightarrow{\mathrm{ID}}| \cdot \cos \left(2 \alpha-\theta_{0}\right)+D_{y}
$$

According to the parametric design of the optical path, the main parameters in the system include angle $\theta_{0}$ of the principal axis, location of the lens $u$, and focal length of the lens $f$. Only when the configurations suffice for all the constraint equations deduced above, will the design requirement be met.

2.3. Model of the Object Depth and Its Corresponding Image Point. When the discrete rotational symmetric triangulation system is applied to measure different depth of object point, the position of the image point on the obtained image is different. In other words, there is a certain relation between the depth of the object point and the position of the image point. According to the parametric design of the optical system mentioned above, the relation between the depth of object point and the position of image point is shown in Figure 4.

The relation model shown in Figure 4 is based on the measuring coordinate system of the system. It can be considered that Z-axis in the machine coordinate system is 


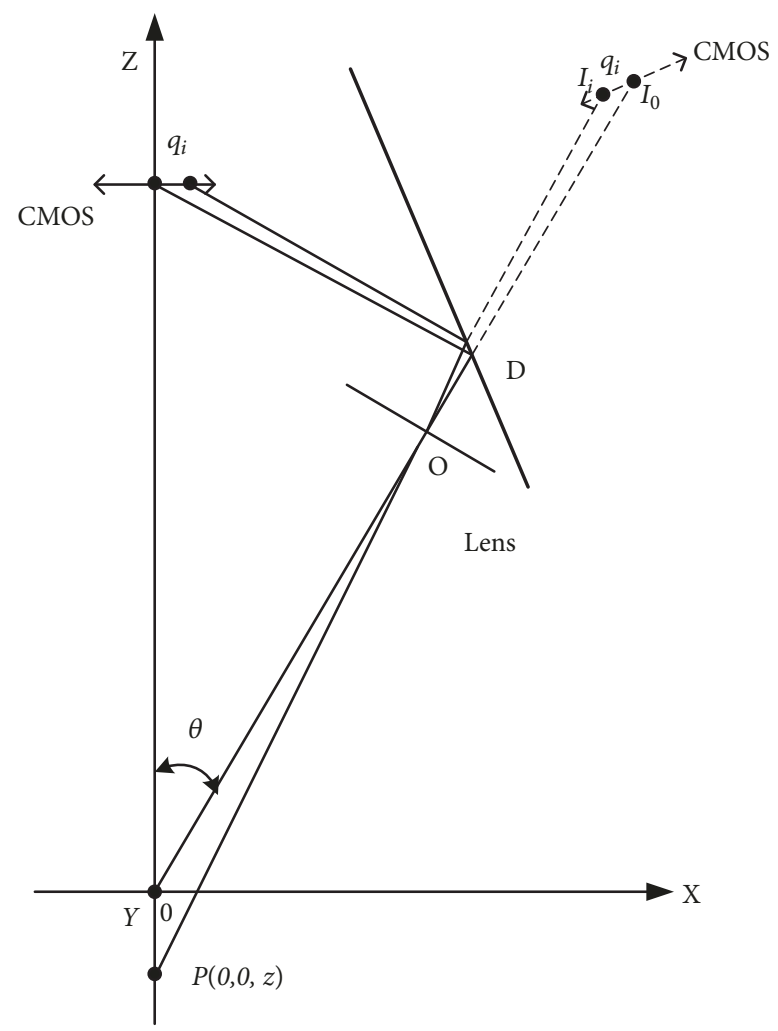

FIgURE 4: Relation between the depth of object point and the position of image point.

the up-and-down moving direction, and the direction where the depth of the object point changes. X-axis is the leftand-right moving direction of the system while $\mathrm{Y}$-axis is the forward-and-backward moving direction. Let arbitrary object point be $\mathrm{P}(0,0, z)$, indicating the depth of the object point. The image point detected on the CMOS camera is $\mathrm{I}_{i}$ and its coordinate on the image is $(u, v)$. The coordinate of the initial original point is $\mathrm{P}(0,0,0)$ and its corresponding image point is $\mathrm{I}_{0}$.

When the object point moves a certain distance in the $\mathrm{Z}$-axis direction, the image point correspondingly moves a certain distance. The position of the image point in the $\mathrm{X}-\mathrm{Z}$ coordinate is $\mathrm{I}(x, z)$, and the offset distance between it and the initial image point $\mathrm{I}_{0}(x, z)$ is $q=\sqrt{\left(I_{u}-I_{0 u}\right)^{2}+\left(I_{v}-I_{0 v}\right)^{2}}$; then

$$
\begin{aligned}
& I_{x}=I_{0 x}+q \sin (\operatorname{dir} \overrightarrow{\mathrm{GO}}) \\
& I_{z}=I_{0 z}+q \cos (\operatorname{dir} \overrightarrow{\mathrm{GO}})
\end{aligned}
$$

According to the object-image positional relation of the ideal optical system, the light beam incidents from the front principal point of the front principal plane and is emitted through the back principle point of the back principal plane. Image point $\mathrm{I}$ and principal point $\mathrm{O}$ are on the same straight line while object point $\mathrm{P}$ and principal point $\mathrm{O}$ are on the same straight line. The slopes of the two straight lines are equal, satisfying the following constraint equation:

$$
\frac{I_{z}-O_{z}}{I_{x}-O_{x}}=\frac{O_{z}-P_{z}}{O_{x}}
$$

The equation in terms of point $\mathrm{P}$ is

$$
P_{z}=O_{z}-\frac{I_{y}-O_{z}}{I_{x}-O_{x}} O_{x}
$$

According to the parameters of the optical system, the theoretical relation model of the system can be obtained; that is, the theoretical equation in terms of the relative distance relation between the depth of object point $\mathrm{P}$ and the image point is

$$
P_{z}=u \cos \theta_{0}-\frac{u \cos \theta_{0}+q \cos d i r \overrightarrow{G O}}{u \sin \theta_{0}+q \sin \operatorname{dir} \overrightarrow{G O}} \cdot u \sin \theta_{0}
$$

According to the size of the CMOS, the value range of $q$ is -3.4 $\mathrm{mm} \sim 3.4 \mathrm{~mm}, u=75 \mathrm{~mm}$; transforming the formula, then the theoretical equation can be rewritten as

$$
\begin{aligned}
& P_{z} \\
& =\frac{\left(u \cos \theta_{0} \sin d i r \overrightarrow{G O}-\cos d i r \overrightarrow{G O} u \sin \theta_{0}\right) q+0}{u \sin \theta_{0}+q \sin \operatorname{dir} \overrightarrow{G O}}
\end{aligned}
$$

Due to the influence of the existent error in machining, assembly processing, and the optical system, the relative distance relation between the depth of point $\mathrm{P}$ and the image point does not conform to the prototype model of the measurement system. Therefore, on the basis of (28), the internal parameters of the prototype of the system are obtained by calibration experiment. Equation (28) can be transformed to give

$$
P_{z}=\frac{A+B q}{C+D q}
$$

Transforming (29) to the form as $\mathbf{A} x=0$, we can obtain

$$
\left[\begin{array}{llll}
-1 & -q & P_{z} & q P_{z}
\end{array}\right]\left[\begin{array}{c}
A \\
B \\
C \\
D
\end{array}\right]=0
$$

After obtaining the multiple sets of $\left(P_{i Z}, q_{i}\right)$, the least square method is adopted to obtain values of the internal parameters $A, B, C, D$ of the system principle prototype. Consequently, (30) is transformed as

$$
\left[\begin{array}{cccc}
-1 & -q_{1} & P_{1 z} & q_{1} P_{1 z} \\
-1 & -q_{2} & P_{2 z} & q_{2} P_{2 z} \\
\vdots & & & \vdots \\
-1 & -q_{i} & P_{i z} & q_{i} P_{i z}
\end{array}\right]\left[\begin{array}{c}
A \\
B \\
C \\
D
\end{array}\right]=0
$$


Therefore, according to (31), the calibration experiment obtains data of multiple sets of $u i, v i, \mathrm{Z}_{w} i$, forming $i \times 4$ matrix $\left[\begin{array}{ccccc}-1 & -q_{1} & P_{1 z} & q_{1} P_{1 z} \\ -1 & -q_{2} & P_{2 z} & q_{2} P_{2 z} \\ \vdots & & & \vdots \\ -1 & -q_{i} & P_{i z} & q_{i} P_{i z}\end{array}\right]$. Conducting the matrix A with singular value decomposition (SVD) and adding constraint condition $\|x\|=1$, the minimum least square solutions are obtained; then the internal parameters $\left[\begin{array}{llll}A & B & C & D\end{array}\right]^{\mathrm{T}}$ of the system are determined. Using formula (28) and the known pixel coordinates $u_{i}, v_{i}$, we can obtain the corresponding $P_{i z}$.

The center-of-gravity method is used to extract the laser spot center in the paper. Assuming that the size of the acquired image is $x \times y$ pixels and letting $B(i, j)$ be for the gray value of the $i t h$ row and $j$ th column pixel, the center-ofgravity $(x, y)$ is calculated as follows.

$$
\begin{gathered}
x=\frac{\sum_{i=1}^{X} \sum_{j=1}^{Y} B[i, j] \times i}{\sum_{i=1}^{X} \sum_{j=1}^{Y} B[i, j]} \\
y=\frac{\sum_{i=1}^{X} \sum_{j=1}^{Y} B[i, j] \times j}{\sum_{i=1}^{X} \sum_{j=1}^{Y} B[i, j]}
\end{gathered}
$$

In the system, the original image information collected by the CMOS contains multiple light spots, and the centerof-gravity method is aimed to compute center in a single spot area. Before using the center-of-gravity method, we need to preprocess the original image with a serial of operations including binarization, open operation, and connected components labeling. Binarization converts 251 original images to images with pixels of only two luminance value (0 and 255). Open operation can make the profile of the spots smooth. After labeling the white pixels with connected components labeling, each individual connected component is formed as an identified block. For the purpose of reducing noise on the basis of the original image, separating, and marking the multiple spots, we conducted the operations above and therefore obtained clear contour and spots center for the further processing.

\section{Simulation}

3.1. Imaging Simulation of Optical Path. The known laser wavelength of the measurement system is $633 \mathrm{~nm}$ and the power is $1 \mathrm{~mW}$. The resolution ratio of the CMOS camera is $1280 \times 1024$ pixels and the pixel size is $5.3 \mu \mathrm{m}$. The focal length of the aspherical plano-convex lens is $37.5 \mathrm{~mm}$; the clear aperture is $25 \mathrm{~mm}$. The focal length of the focusing lens is $72 \mathrm{~mm}$. The diameter of the mirror is $25 \mathrm{~mm}$. The surface of the object is Lambertian's surface, which is the ideal diffuse surface. Considering the feasibility of placing multiple lens, mirrors, lasers, and cameras in the internal structural space of the system, we determined the principal optical axis angle for $\theta_{0}=20^{\circ}$ as well as the optical systematic parameters in Table 1. The unmarked unit in the blank is $\mathrm{mm}$.

Input the system parameters into Zemax software and conduct imaging simulations for the optical paths. The simulation of the system is shown in Figure 5. The emergent laser is focused on the object surface and diffuse reflection
TABLE 1: Parameters of the optical system.

\begin{tabular}{lccc}
\hline Parameter & Value & Parameter & Value \\
\hline$u$ & 75 & $\mathrm{D}$ & $(37.613,103.341)$ \\
$\alpha$ & $45^{\circ}$ & $\mathrm{P}$ & $(51.303,140.954)$ \\
$\beta$ & $70^{\circ}$ & $h$ & 117.031 \\
$\operatorname{dir} \overrightarrow{\mathrm{GO}}$ & $40^{\circ}$ & $\mathrm{E}$ & $(0,79.813)$ \\
$\mathrm{O}$ & $(25.652,70.477)$ & $\left|\mathrm{DP}^{\prime}\right|$ & 40.027 \\
\hline
\end{tabular}

occurs. Part of the scattered light is imaged on the receiving camera from five different directions through the receiving optical paths.

The imaging of the receiving camera was simulated by the detector module under the nonsequential mode in the Zemax software. Assuming that the distance between the measured object and the origin of the system is $-3 \mathrm{~mm}$, the imaging condition of the receiving camera is shown in Figure 6. In Figure 6(a), five elliptical annular spots are formed on the receiving camera and each consists of a focal point and an elliptical ring. The focal point is the image point formed by the optical system, and the elliptical ring is formed by dispersed speckles. Due to the optical structural characteristics of the discrete rotational symmetric triangulation system, the receiving camera forms five light spots with the same shape, which are discrete circular uniformly distributed around the geometrical center of the image plane of the receiving camera. Figure 6(b) shows the distribution of light intensity on the dotted line in Figure 6(a), which passes through the right elliptical annular light spot. The gray profile of the right elliptical annular spot is approximate to the Gaussian distribution. The noncoherent illumination of the focal point is about $0.035 \mathrm{~W} / \mathrm{cm}^{2}$, and the noncoherent illumination of the elliptical ring is about $0.007 \mathrm{~W} / \mathrm{cm}^{2}$.

According to the depth variation of the object, the imaging condition of the receiving camera is different. When the measured distance between the measured object and the origin of the system is, respectively, $0 \mathrm{~mm}$ and $3 \mathrm{~mm}$, the imaging results on the receiving camera are shown in Figures 7 and 8. Figures 7(b) and 8(b) were obtained similarly to Figure 6(b). As is shown in Figure 7, at $0 \mathrm{~mm}$ position, five oval-shaped light spots formed from different directions converge, whose focal points overlap at one point. As is shown in Figure 8, at $3 \mathrm{~mm}$ position, the position of the elliptical annular spots changes but still owns discrete circumferential array characteristics.

3.2. Simulation about Different Surface Characteristics. The surface characteristics of different objects affects the imaging of the system. Compared with the traditional laser triangulation, the discrete rotational symmetric triangulation system has the advantage that its imaging has rotational symmetry and is less affected by the surface properties of the object. Surface properties of the object vary widely; we chose two kinds of surface material: the sprayed white surface similar to the standard diffuse reflection and the metal surface milled by machine tool which is between the ideal diffuse reflection and specular reflection. 


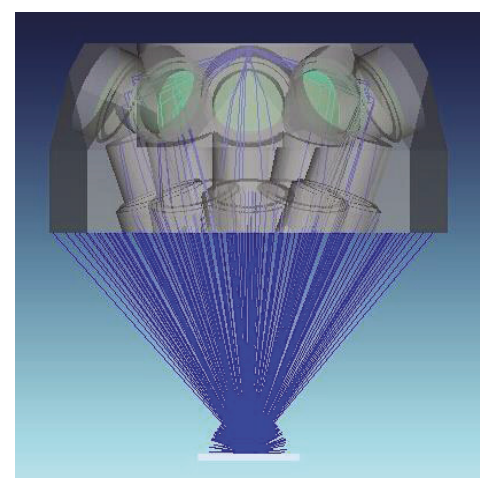

(a)

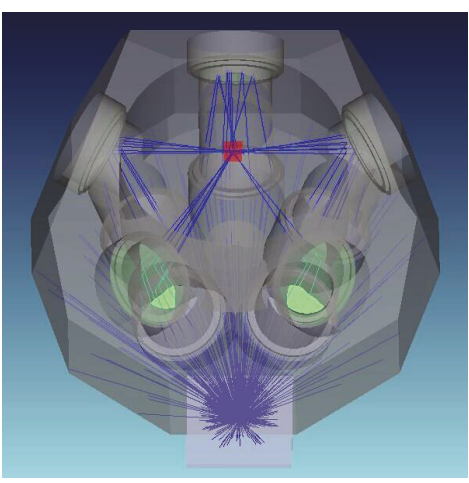

(b)

FIGURE 5: Simulation of the optical system.

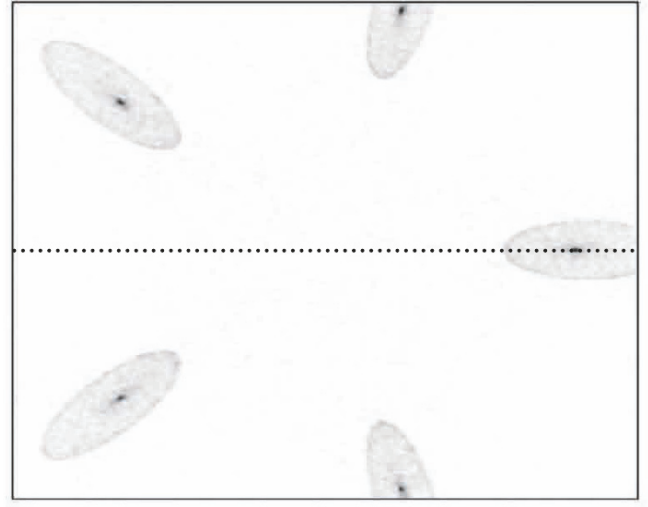

(a)

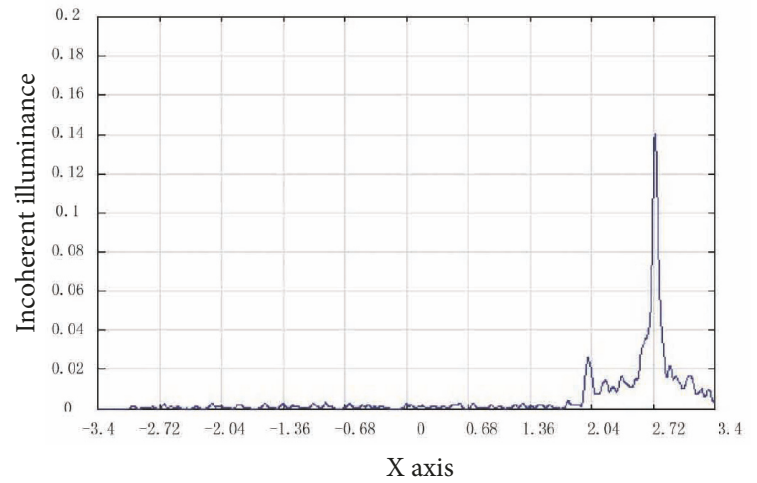

(b)

FIGURE 6: Imaging condition of receiving camera at $-3 \mathrm{~mm}$ position.

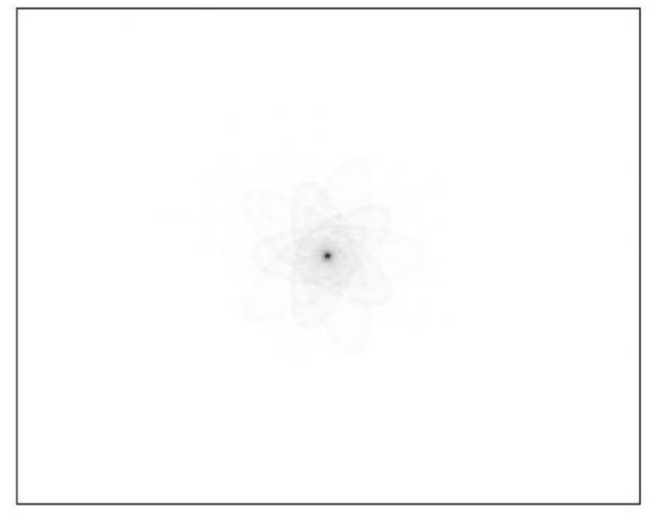

(a)

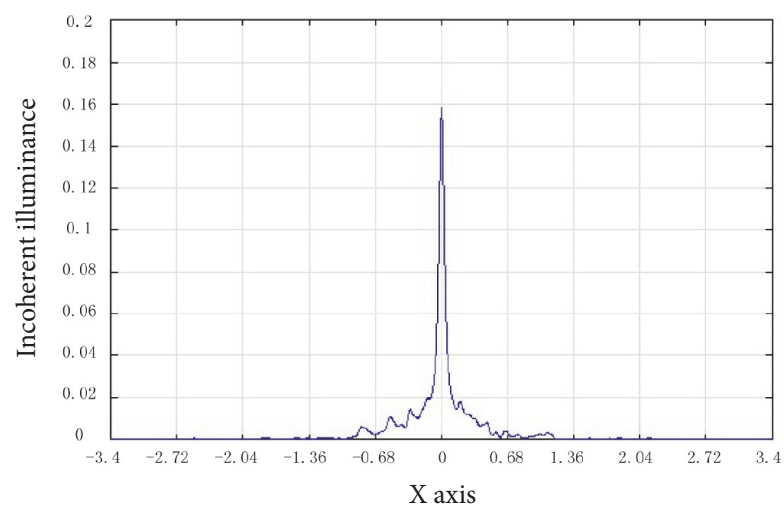

(b)

FIGURE 7: Imaging condition of receiving camera at $0 \mathrm{~mm}$ position. 


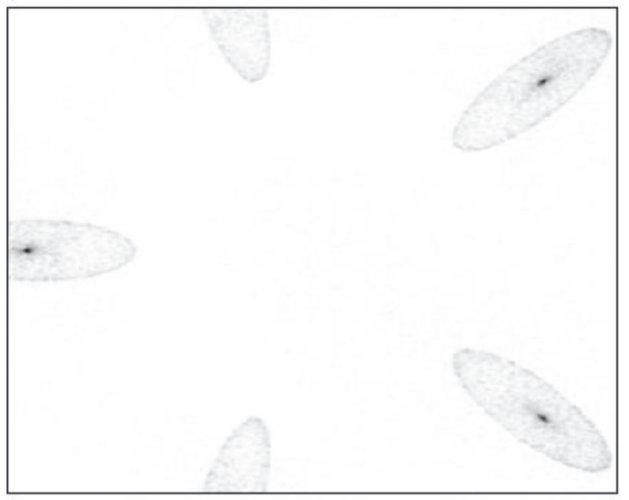

(a)

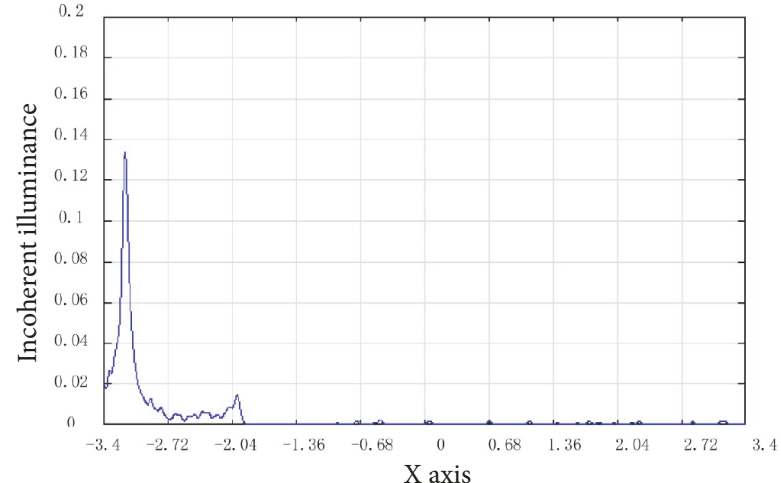

(b)

FIGURE 8: Imaging condition of receiving camera at $3 \mathrm{~mm}$ position.
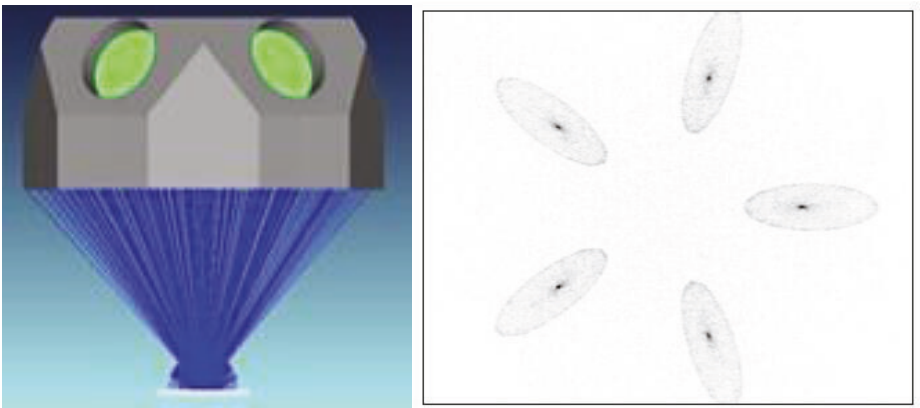

(a)
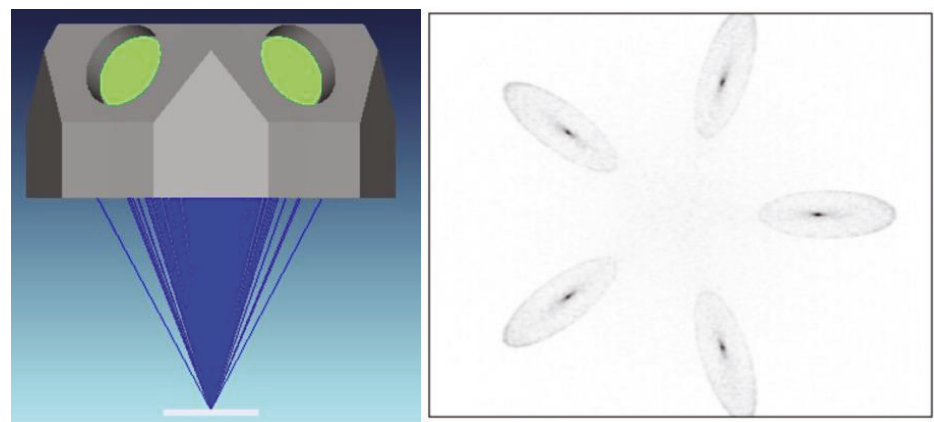

(b)

Figure 9: Simulation of different surface texture. (a) Diffuse reflection surface. (b) Metal surface.

In the Zemax software, we set the distance between the measured object and the origin of the measurement system for $-2 \mathrm{~mm}$. Surface characteristics of measured surface will greatly affect imaging performance; thus we analysed 3 aspects of surface characteristics including surface texture, inclination degree, and existence of occlusion. In the simulation of surface texture, we used the sprayed white surface and the metal surface. The normal direction of the measured surface is parallel to the laser axis of the measuring system, which is shown in Figure 9. Figure 9(a) is the imaging condition of the standard diffuse reflection; the object points are clear and the outlines of the oval-shaped rings are uniform. Figure 9(b) is the imaging condition of the metal surface, the scattered light is less visible compared with the diffuse reflection surface; the object is clearly imaged, but the elliptical annular profile is not uniform.

Simulation of inclination degree is shown in Figure 10. Imaging on the receiving camera is different as the inclination degree is different. Figures $10(\mathrm{a})$ and 10(b) represent the imaging on the receiving camera with diffuse reflection surface. The inclination angle in Figure $10(\mathrm{a})$ is $5^{\circ}$ while it is $60^{\circ}$ in Figure 10(b). Figures $11(\mathrm{a})$ and $11(\mathrm{~b})$ are the imaging with metal surface. The inclination angle in Figures 11(a) and 11(b) are, respectively, $5^{\circ}$ and $15^{\circ}$. 

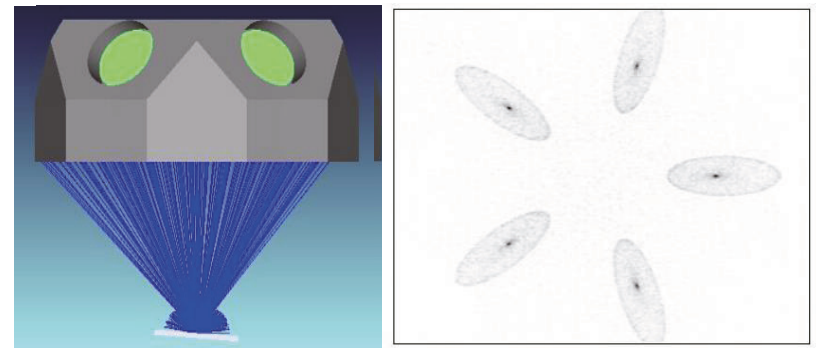

(a)

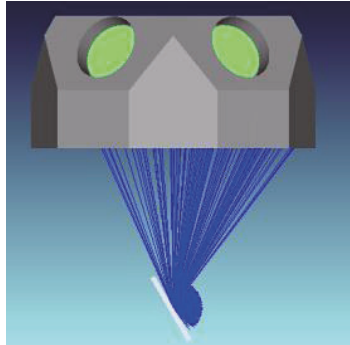

(b)

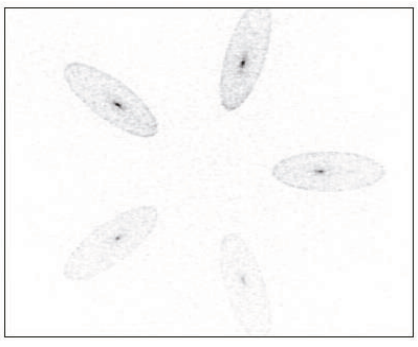

Figure 10: Simulation of diffuse reflection surface with different inclination angle. (a) $5^{\circ}$ and (b) $60^{\circ}$.
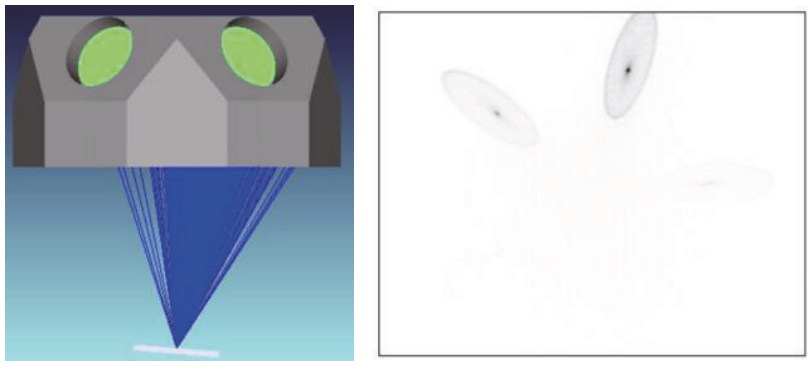

(a)

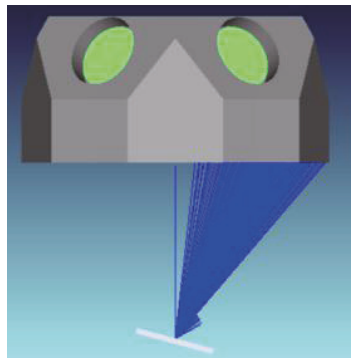

(b)

FIGURE 11: Simulation of metal surface with different inclination angle. (a) $5^{\circ}$ and (b) $15^{\circ}$.
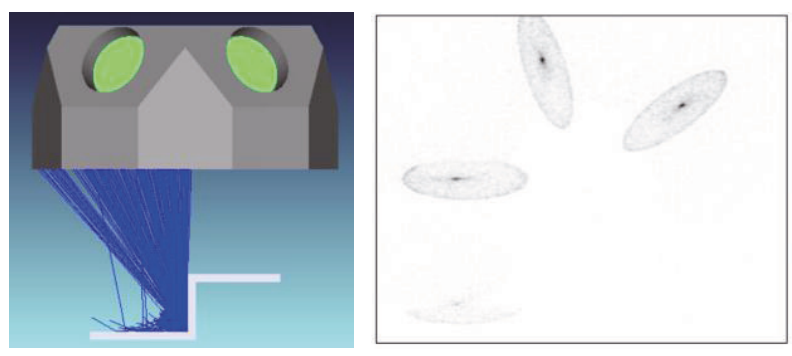

(a)
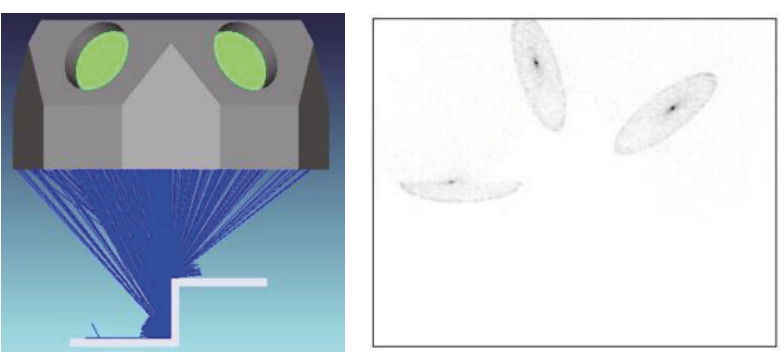

(b)

FIGURE 12: Simulation of occlusion condition. (a) Hit on the lower surface. (b) Hit at the critical position.

Simulation of occlusion condition is as follows: when a portion of light scattered from the measured surface is blocked, the receiving camera will receive incomplete elliptical annular spots. As shown in Figure 12, the step surface is the diffuse reflection surface. Figure 12(a) shows the imaging condition when the laser hits the lower surface. Due to the occlusion effect of the step surface, lights scattered by the lower surface hit the vertical plane and eventually produce an erroneous spot, which is shown as the left bottom spot in Figure 12(a). The laser in Figure 12(b) is exactly at the critical position, hitting partly on the lower surface and partly on the upper surface, leaving only two integrated spots and another half spot.

According to the simulations of different surface characteristics presented above, the discrete rotational triangulation system can detect at least one clear spot; thus information contained in these light spots can be used for the further calculation. Therefore, it is no longer a serious problem with this probe that the directional dependency of traditional laser triangulation may fail to receive light spots.

\section{Experiment}

A measuring system was constructed for a series of experiments in this section. As shown in Figure 13, it is composed of prototype, computer, and CNC machine. Figure 14 shows the mechanical construction of the optical probe, which consists of receiving lens, mirror, receiving camera, and laser.

The receiving lens and the mirror were fixed by a pressing ring. The focusing lens was mounted in a base with an external thread and can be adjusted up and down to ensure that the laser is well focused. The laser was installed in the lens base and it has a certain contact area with the base to ensure the laser is vertical down instead of moving around. Two batteries were installed for providing power. CMOS camera was fixed on the camera stand, which can also be 


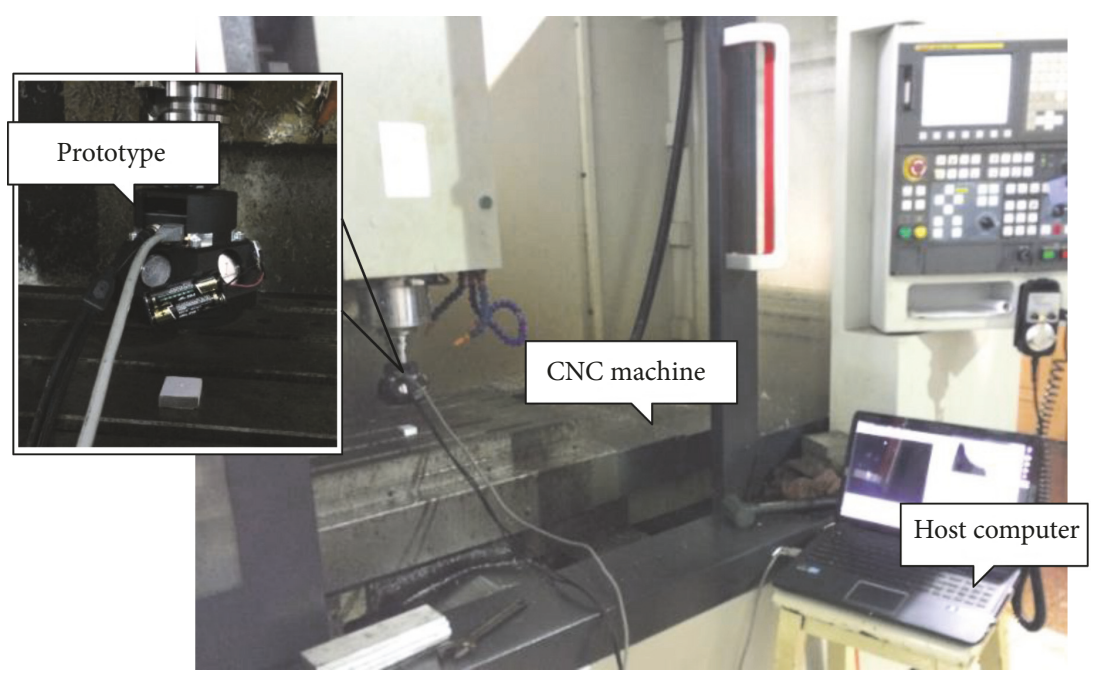

FIGURE 13: Construction of measuring system.

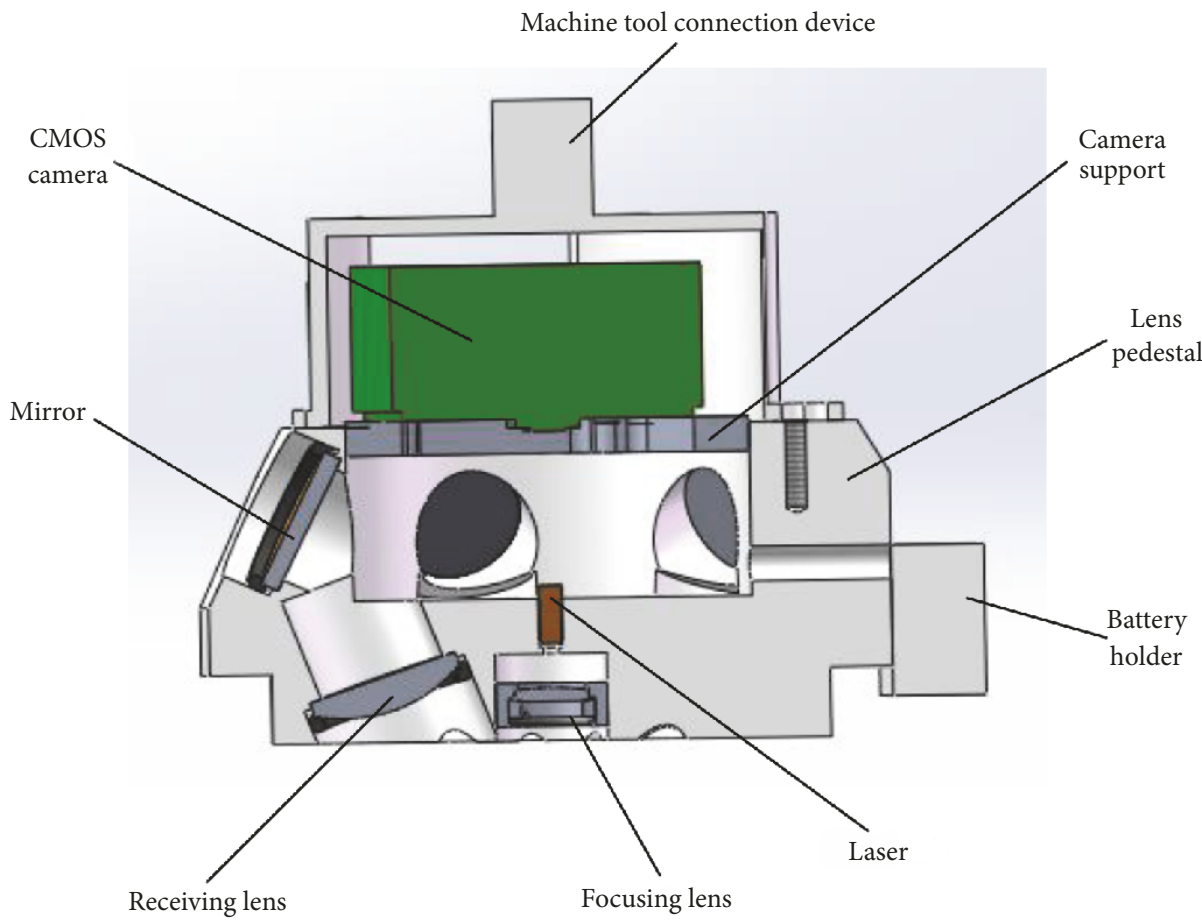

FIGURE 14: Mechanical construction of prototype.

adjusted up and down to ensure good imaging of the light spots. To connect with CNC machines, a machine connection device was designed, whose diameter is $16 \mathrm{~mm}$. The prototype device used in the experiment is shown in Figure 15. The measuring range of the system is $\pm 3 \mathrm{~mm}$ and the working distance is $63.66 \mathrm{~mm}$. The system enables capturing 50 images per second with the help of the $50 \mathrm{fps}$ CMOS camera.

4.1. The Accuracy of Laser Spot Detection Method. In this experiment, the center-of-gravity method, which is adopted in our system, is compared with the peak subpixel method.
There are three commonly used algorithms of the peak subpixel method, including moment estimate, interpolation, and fitting. Compared with the former two algorithms, fitting algorithm is insensitive to noise and owns good stability. Therefore, the fitting algorithm was employed while using peak subpixel method. Image A in Figure 16 is the original image acquired by the CMOS, which is shown in Figure 17(a). A series of operations are processed to reduce noise of the original image, including binarization, open operation, and connected components labeling; then image B is obtained. The grayscale of image B is 0 and 1 and each marked spot is even and symmetrical, as is shown in Figure 17(b). The image 


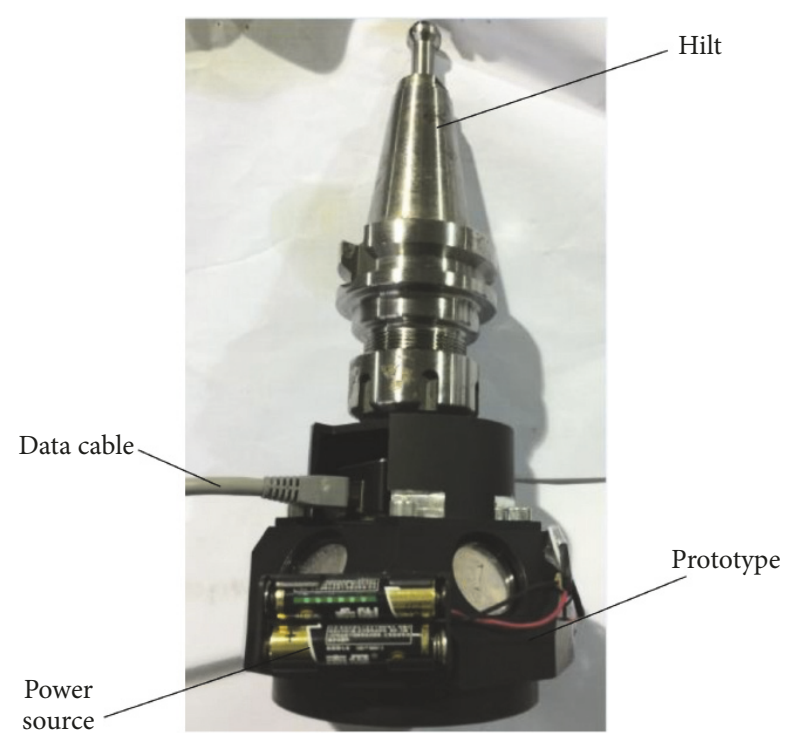

FIGURE 15: Actual structure of prototype.

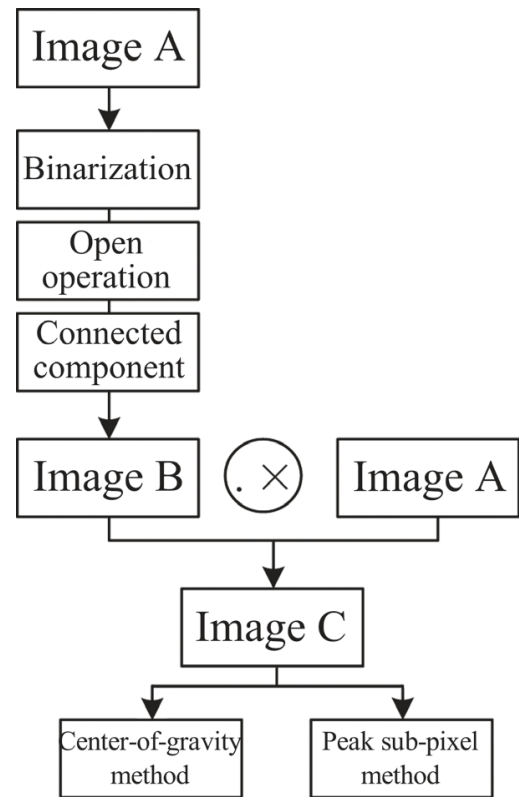

FIGURE 16: Flow chart of extracting spot center using two methods.

C is obtained after dot production of image B and image A. Each spot in image $\mathrm{C}$ is a grayscale image with 256 levels.

According to the pixel coordinate $(i, j)$ of each spot obtained by connected components labeling, the coordinate $(x, y)$ of the spot center can be found referring to (32) and (33). The results of extracting the center of spots using center-of-gravity method and the peak subpixel method are, respectively, shown in Figure 18.

We used the image of white paper $0^{\circ}$ and height $1 \mathrm{~mm}$ to compare the two methods. After capturing 20 images of the five spots, we obtained 100 spots for calculation of the repeatability accuracy of the system. The repeatability accuracy $S$ of the two methods is shown in Figure 19 and
TABLE 2: Repeatability accuracy $S$ of the white paper $0^{\circ}$. Unit:Pixel.

\begin{tabular}{lccccc}
\hline White paper $0^{\circ}$ & P1 & P2 & P3 & P4 & P5 \\
\hline Peak sub-pixel & 2.09956 & 1.54522 & 1.07046 & 1.46535 & 2.06641 \\
Center-of-gravity & 0.13487 & 0.24098 & 0.20842 & 0.23199 & 0.17006 \\
\hline
\end{tabular}

Table 2. The peak subpixel method is not stable and its repeatability accuracy of each spot is not as good as the center-of-gravity method.

Besides the repeatability accuracy, we also compared the two methods by analysing the trajectory of a certain image point. Specifically, we measured the coordinates of several groups of image points and then fit them into a straight line to observe the distribution of the distance between each image point and the straight line. Figure 20 shows the trajectory of the image point P1 on the CMOS. The center of the spot is extracted, respectively, by the centerof-gravity method and the peak subpixel method. The red line indicates the fitting line. From Figure 20, we can see that almost all the spots extracted by the center-of-gravity are on the straight line, which shows good linearity. The center of the spots extracted by the peak subpixel method distribute dispersively, which shows poor linearity. It shows that the center-of-gravity method is more stable than the peak subpixel method in terms of the spot center extraction algorithm.

4.2. Calibration Experiment. After obtaining the spot centers by the center-of-gravity method, we conducted a linear calibration experiment by the center-of-gravity method. We installed system prototype to the 3 -axis $\mathrm{CNC}$ machine by handle connection and used numerical control system to control the measurement system moving up and down. When the spot becomes minimum, the current machine relative $\mathrm{Z}$ coordinate is saved, which is set to the original point of the probe. Then, the system height is adjusted for conducting calibration. Figure $21(\mathrm{a})$ is the site calibration picture, Figure 21(b) is the image information obtained by the system, and Figure 21(c) is the machine tool coordinate system of the current system displayed by CNC machine tool, which can also be regarded as the world coordinate system. $P_{z}$ is 0 . Under the condition of white paper surface $0^{\circ}$, points were captured every $0.1 \mathrm{~mm}$ of the total measuring range ($3 \mathrm{~mm}$ to $3 \mathrm{~mm}$ ); thus 61 sets of points were obtained for calibration.

The data $u, v$, and $P_{z}$ calibrated under the condition of the white paper surface $0^{\circ}$ are brought into the matrix of (31); after the singular value decomposition, the least square solution of the internal parameters $\left[\begin{array}{llll}A & B & C & D\end{array}\right]^{\mathrm{T}}$ of the prototype can be obtained; that is,

$$
\left[\begin{array}{l}
A \\
B \\
C \\
D
\end{array}\right]=\left[\begin{array}{c}
-0.005697 \\
25.623679 \\
25.664288 \\
0.639041
\end{array}\right]
$$




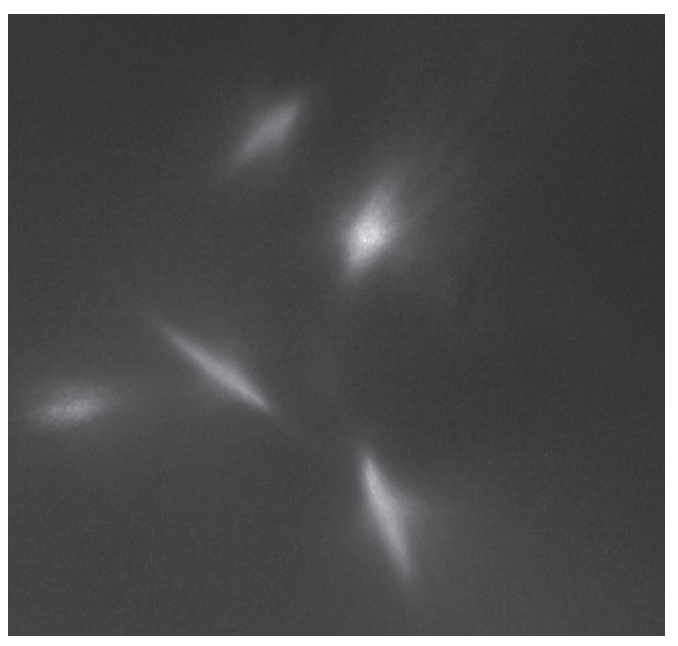

(a) Original image

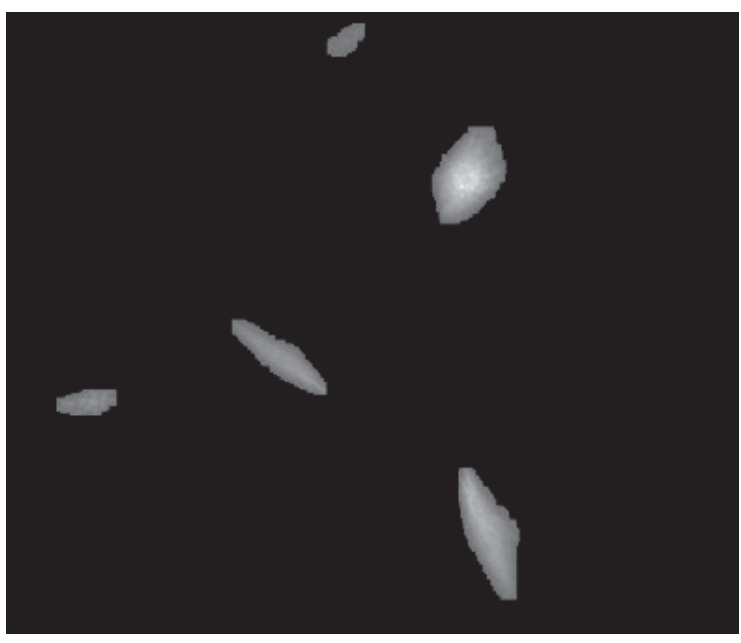

(b) Greyscale image after noise reduction

FiguRE 17: Images before and after noise reduction.

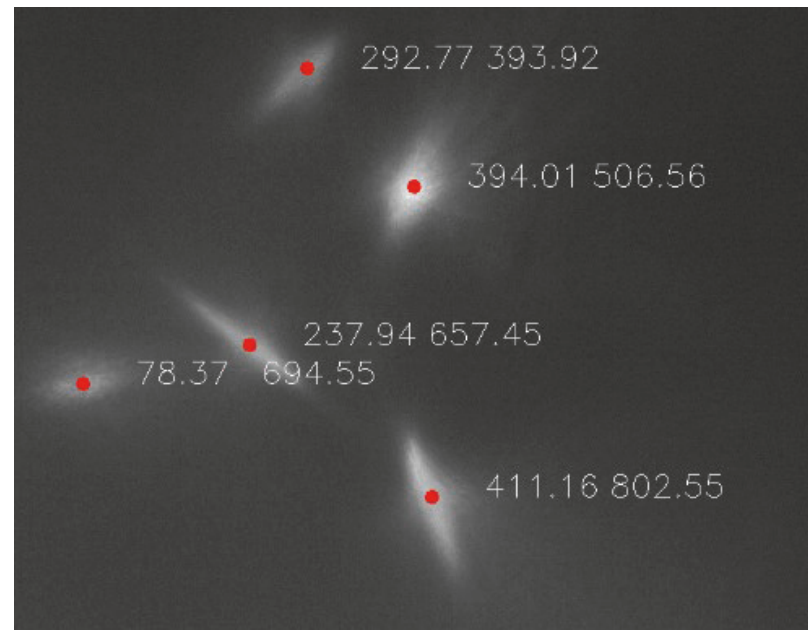

(a) Center-of-gravity method

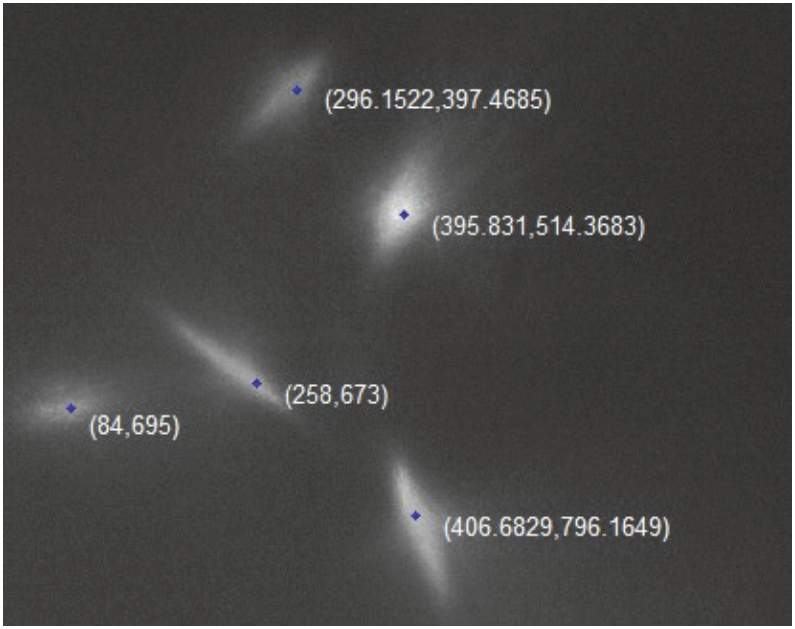

(b) Peak subpixel method

FIGURE 18: Extracting result of the two methods.

Substituting it into (29), the relational expression of the object point depth $P_{z}$ can be obtained as

$$
P_{z}=\frac{-0.005697+25.623679 q}{25.664288+0.639041 q}
$$

4.3. Performance of the Optical Probe. In this section, performance of the optics in several aspects was investigated and values of several essential items such as repeatability accuracy, resolution, linearity, and sensitivity of the optics are provided. The evaluation results were obtained strictly by conducting controlled experiments under different conditions.

For the purpose of obtaining the repeatability accuracy of the system, we conducted repeatability experiment of single point. The single point repeatability experiment is divided into 8 classes by surface characteristics: white paper surface $0^{\circ}$, metal surface $0^{\circ}$, white paper surface $30^{\circ}$, metal surface

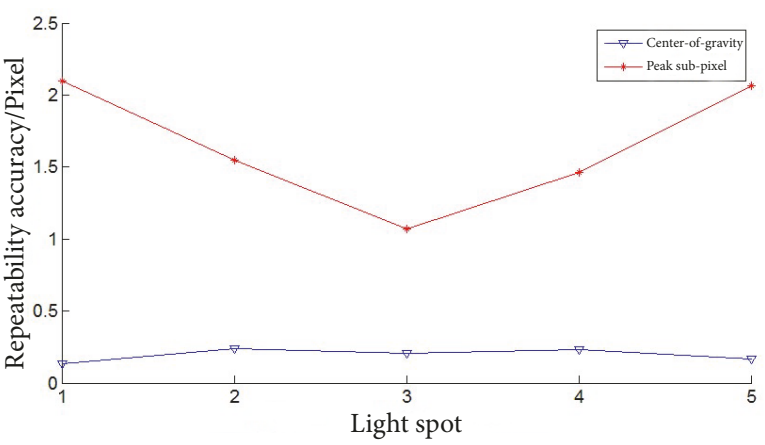

FIGURE 19: Comparison of the repeatability of two methods.

$30^{\circ}$, white paper surface $45^{\circ}$, metal surface $45^{\circ}$, white paper surface $60^{\circ}$, and metal surface $60^{\circ}$. The data collected 20 times at the same position of each class is regarded as a group. The 


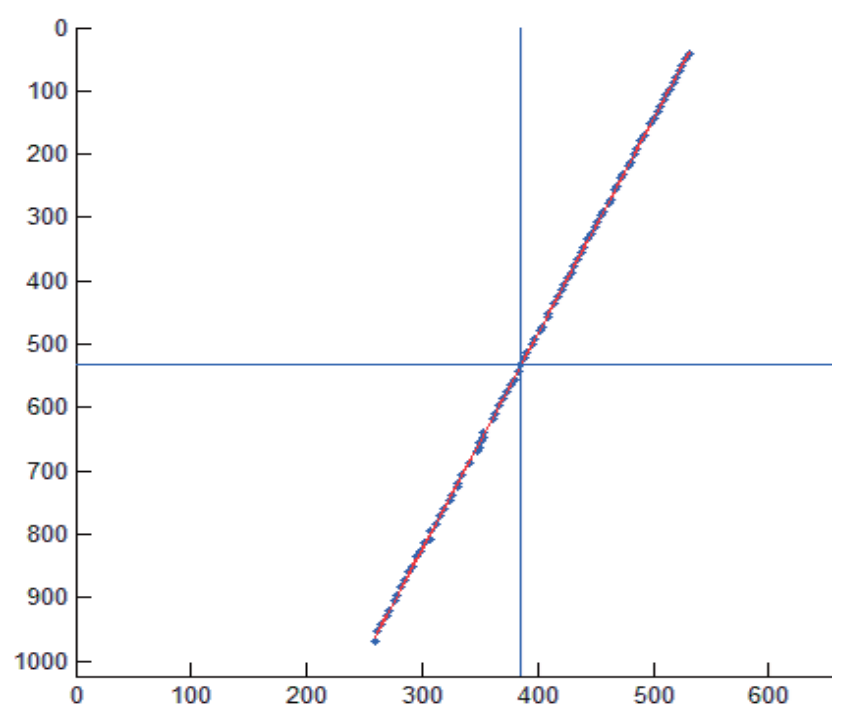

(a)

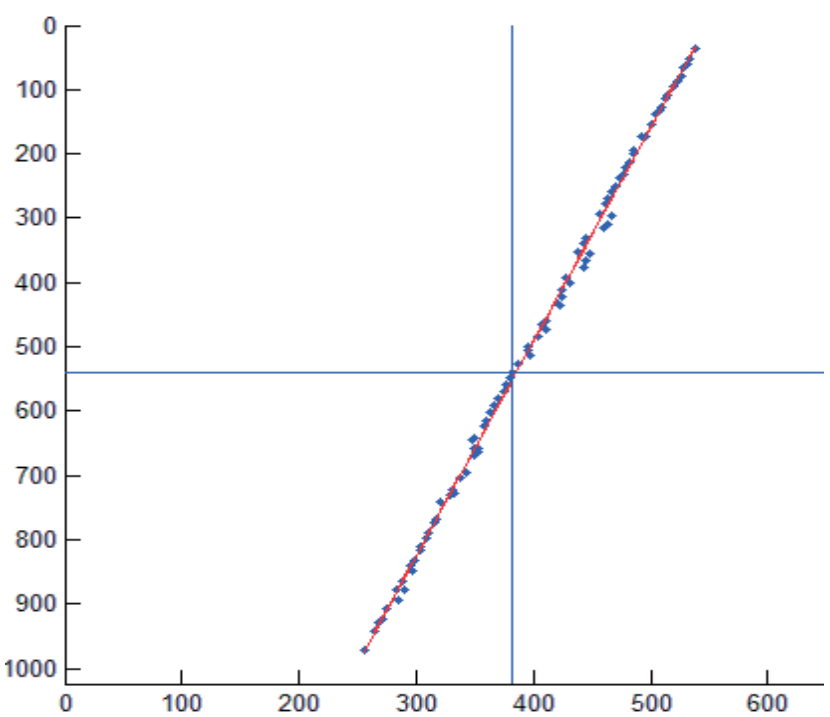

(b)

FIgURE 20: Trajectory of the image point P1 of the CMOS. (a) The center-of-gravity method. (b) The peak subpixel method.

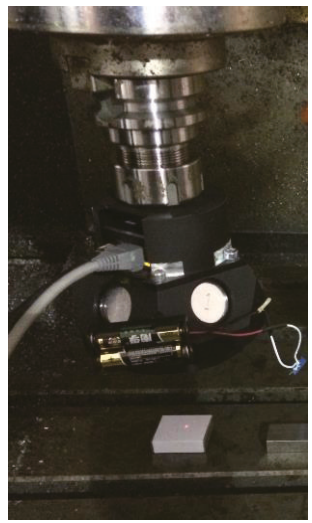

(a)

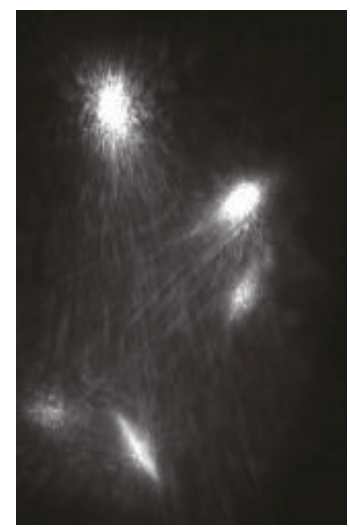

(b)

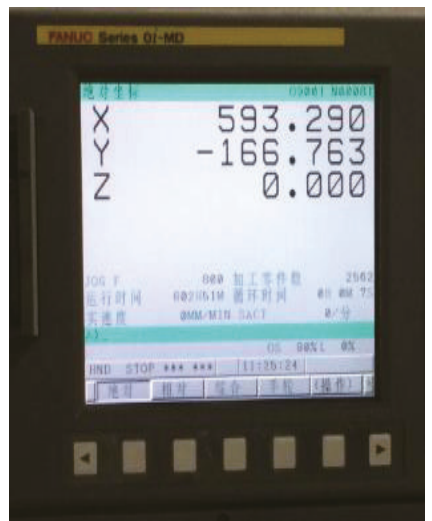

(c)

FIGURE 21: Calibration experiment.

standard deviation was computed for each set of data; then the repeatability accuracy of the measurement system $S_{n}(i)$ can be obtained as

$$
\begin{aligned}
d_{i-j} & =\sqrt{\left(x_{i-j}-\text { mean }\left(x_{i}\right)\right)^{2}+\left(y_{i-j}-\text { mean }\left(y_{i}\right)\right)^{2}} \\
S_{n}(i) & =\operatorname{std}\left(d_{i-j}\right)
\end{aligned}
$$

where $d_{i-j}$ represents the deviation between the $j$ th data at the ith height and the mean value of data at the ith height. $S_{n}$ represents the repeatability accuracy of different surface characteristics.

In each class of the eight, we repeatedly measured an identical point 20 times, at the position whose relative height is, respectively, $1 \mathrm{~mm}, 0.5 \mathrm{~mm}, 0 \mathrm{~mm},-0.5 \mathrm{~mm}$, and $-1 \mathrm{~mm}$. The surface characteristic is divided into two kinds, which is the standard white paper surface approximately to the standard diffuse reflection surface and the metal surface between specular reflection and diffuse reflection surface. The angle of the tilted surface is, respectively, set to $0^{\circ}, 30^{\circ}, 45^{\circ}$, and $60^{\circ}$.

Considering that each image obtained by CMOS camera contains 5 light spots, the repeatability of the 5 light spots is also required to be investigated. The five spots are named in order as P1, P2, P3, P4, and P5. The measurement result of the repeatability accuracy is shown in Tables 3-10. The data in the tables is obtained by the center-of-gravity method. The unit is pixel. The known size of the pixel is $5.3 \mu \mathrm{m}$.

From the data in Tables 3-10, the repeatability accuracy of spots detection is less than $5.3 \mu \mathrm{m}$. The overall repeatability accuracy can be defined as the average of all the data, which is $\overline{\text { all }}=0.13611$ pixel. Thus, the overall repeatability accuracy is calculated as about $0.72 \mu \mathrm{m}$. 
TABLE 3: Repeatability accuracy $S_{W 0}$ of the white paper $0^{\circ}$ for light spots detection. Unit: Pixel.

\begin{tabular}{lccccc}
\hline White paper $0^{\circ}$ & $-1 \mathrm{~mm}$ & $-0.5 \mathrm{~mm}$ & $0 \mathrm{~mm}$ & $0.5 \mathrm{~mm}$ & $1 \mathrm{~mm}$ \\
\hline P1 & 0.16715 & 0.20355 & 0.07016 & 0.23691 & 0.13487 \\
P2 & 0.20069 & 0.22597 & 0.05861 & 0.20332 & 0.24098 \\
P3 & 0.35130 & 0.18276 & 0.03523 & 0.22946 & 0.20842 \\
P4 & 0.20807 & 0.16927 & 0.12157 & 0.14101 & 0.23199 \\
P5 & 0.29075 & 0.20208 & 0.19424 & 0.20051 & 0.17006 \\
\hline
\end{tabular}

TABLE 4: Repeatability accuracy $S_{W 30}$ of the white paper $30^{\circ}$ for light spots detection. Unit: Pixel.

\begin{tabular}{lccccc}
\hline White paper $30^{\circ}$ & $-1 \mathrm{~mm}$ & $-0.5 \mathrm{~mm}$ & $0 \mathrm{~mm}$ & $0.5 \mathrm{~mm}$ & $1 \mathrm{~mm}$ \\
\hline P1 & 0.03616 & 0.20174 & 0.19777 & 0.02339 & 0.04682 \\
P2 & 0.01896 & 0.29682 & 0.02136 & 0.01829 & 0.01858 \\
P3 & 0.01701 & 0.31987 & 0.01709 & 0.01228 & 0.01509 \\
P4 & 0.03488 & 0.32332 & 0.19131 & 0.02527 & 0.04095 \\
P5 & 0.03864 & 0.24065 & 0.18674 & 0.01201 & 0.05083 \\
\hline
\end{tabular}

TABLE 5: Repeatability accuracy $S_{W 45}$ of the white paper $45^{\circ}$ for light spots detection. Unit: Pixel.

\begin{tabular}{lccccc}
\hline White paper $45^{\circ}$ & $-1 \mathrm{~mm}$ & $-0.5 \mathrm{~mm}$ & $0 \mathrm{~mm}$ & $0.5 \mathrm{~mm}$ & $1 \mathrm{~mm}$ \\
\hline P1 & 0.13136 & 0.06444 & 0.20803 & 0.07498 & 0.07673 \\
P2 & 0.02951 & 0.05114 & 0.18380 & 0.04482 & 0.05987 \\
P3 & 0.05600 & 0.05727 & 0.26172 & 0.05315 & 0.03151 \\
P4 & 0.11268 & 0.03286 & 0.23092 & 0.14385 & 0.06969 \\
P5 & 0.14806 & 0.04835 & 0.20892 & 0.03599 & 0.09220 \\
\hline
\end{tabular}

TABLE 6: Repeatability accuracy $S_{W 60}$ of the white paper $60^{\circ}$ for light spots detection. Unit: Pixel.

\begin{tabular}{lccccc}
\hline White paper $60^{\circ}$ & $-1 \mathrm{~mm}$ & $-0.5 \mathrm{~mm}$ & $0 \mathrm{~mm}$ & $0.5 \mathrm{~mm}$ & $1 \mathrm{~mm}$ \\
\hline P1 & 0.28931 & 0.21591 & 0.25977 & 0.22617 & 0.26644 \\
P2 & 0.32165 & 0.21035 & 0.14368 & 0.22923 & 0.31022 \\
P3 & 0.20475 & 0.19001 & 0.17399 & 0.21353 & 0.28663 \\
P4 & 0.24432 & 0.27027 & 0.19571 & 0.38182 & 0.31105 \\
P5 & 0.20679 & 0.27710 & 0.28136 & 0.19204 & 0.29698 \\
\hline
\end{tabular}

TABLE 7: Repeatability accuracy $S_{M 0}$ of the metal surface $0^{\circ}$ for light spots detection. Unit: Pixel.

\begin{tabular}{lccccc}
\hline Metal $0^{\circ}$ & $-1 \mathrm{~mm}$ & $-0.5 \mathrm{~mm}$ & $0 \mathrm{~mm}$ & $0.5 \mathrm{~mm}$ & $1 \mathrm{~mm}$ \\
\hline P1 & 0.26173 & 0.04788 & 0.04705 & 0.16207 & 0.05965 \\
P4 & 0.30383 & 0.06944 & 0.07752 & 0.08127 & 0.07647 \\
\hline
\end{tabular}

Afterwards, we conducted accuracy experiments on the calibrated prototype based on (35). We, respectively, measured the white paper surface and the metal surface within the range of $-2 \mathrm{~mm}$ to $2 \mathrm{~mm}$ and obtained its corresponding spot pixel coordinates using the center-of-gravity method. Points were captured every $0.1 \mathrm{~mm}$ within the range; thus 41 sets of points were obtained for both conditions. The pixel coordinates $u, v$ of part of the light spots and the depth $P_{z}$ of the object point under the condition of white paper
TABLE 8: Repeatability accuracy $S_{M 30}$ of the metal surface $30^{\circ}$ for light spots detection. Unit: Pixel.

\begin{tabular}{lccccc}
\hline Metal $30^{\circ}$ & $-1 \mathrm{~mm}$ & $-0.5 \mathrm{~mm}$ & $0 \mathrm{~mm}$ & $0.5 \mathrm{~mm}$ & $1 \mathrm{~mm}$ \\
\hline P4 & 0.02384 & 0.01741 & 0.02909 & 0.0182 & 0.04724 \\
P5 & 0.01671 & 0.01523 & 0.02499 & 0.02569 & 0.04468 \\
\hline
\end{tabular}

TABLE 9: Repeatability accuracy $S_{M 45}$ of the metal surface $45^{\circ}$ for light spots detection. Unit: Pixel.

\begin{tabular}{lccccc}
\hline Metal $45^{\circ}$ & $-1 \mathrm{~mm}$ & $-0.5 \mathrm{~mm}$ & $0 \mathrm{~mm}$ & $0.5 \mathrm{~mm}$ & $1 \mathrm{~mm}$ \\
\hline P4 & 0.02414 & 0.02332 & 0.03160 & 0.02263 & 0.15897 \\
P5 & & 0.01933 & 0.01762 & 0.01290 & \\
\hline
\end{tabular}

TABLE 10: Repeatability accuracy $S_{M 60}$ of the metal surface $60^{\circ}$ for light spots detection. Unit: Pixel.

\begin{tabular}{lccccc}
\hline Metal $60^{\circ}$ & $-1 \mathrm{~mm}$ & $-0.5 \mathrm{~mm}$ & $0 \mathrm{~mm}$ & $0.5 \mathrm{~mm}$ & $1 \mathrm{~mm}$ \\
\hline P1 & 0.04343 & 0.04070 & 0.46055 & 0.03919 & 0.03974 \\
\hline
\end{tabular}

surface $0^{\circ}$ are shown in Figure 22, from which we can see that there is a certain error between the calculated value and the measured value. The standard deviation of the error of the 41 sets of data was calculated and the reference value of the prototype measurement accuracy under the condition of the white paper surface was obtained as $0.0294 \mathrm{~mm}$. Likewise, similar experiment process was conducted for the metal surface. The pixel coordinates $u, v$ of $\mathrm{P} 4$ light spots and the depth $P_{z}$ of the object points under the condition of the metal surface $0^{\circ}$ are shown in Figure 23. The reference value of the prototype measurement accuracy under the condition of the metal surface is obtained by analysing 41 sets of data, which is $0.0399 \mathrm{~mm}$.

Afterwards, we conducted tilted surface experiment. The tilted surface changes the intensity and distribution of the beam in the receiving direction, resulting in an offset of the speckle center in the image plane. The white object and the metal object with the inclination angle of $30^{\circ}, 45^{\circ}$, and $60^{\circ}$ were placed on the machine and measured by the prototype. Figure 24 shows the measurement of the white surface object. The spot imaging does not change much due to the effect of ideal diffuse reflection of the white surface. In actual measurement, the five spots did not change significantly when the angle of inclination changed. However, the brightness of the spots of $60^{\circ}$ had a prominent decline compared with that of $0^{\circ}, 30^{\circ}$, and $45^{\circ}$, owing to a large part of the diffuse reflected lights being blocked.

Metal surface owns the reflection characteristics between diffuse reflection and specular reflection. White paper surface is smooth and it is imaged uniformly, while metal surface is quite the opposite. In addition, spots of the metal surface change significantly as the inclination angle changes. The experiment process is similar to that for the white paper and metal surfaces of $0^{\circ}$ described above. The results for the white surface of $30^{\circ}$ and the metal surface of $30^{\circ}$ are shown in Figures 25 and 26, from which the measurement accuracy is, respectively, calculated as $0.0290 \mathrm{~mm}$ and $0.0357 \mathrm{~mm}$. 


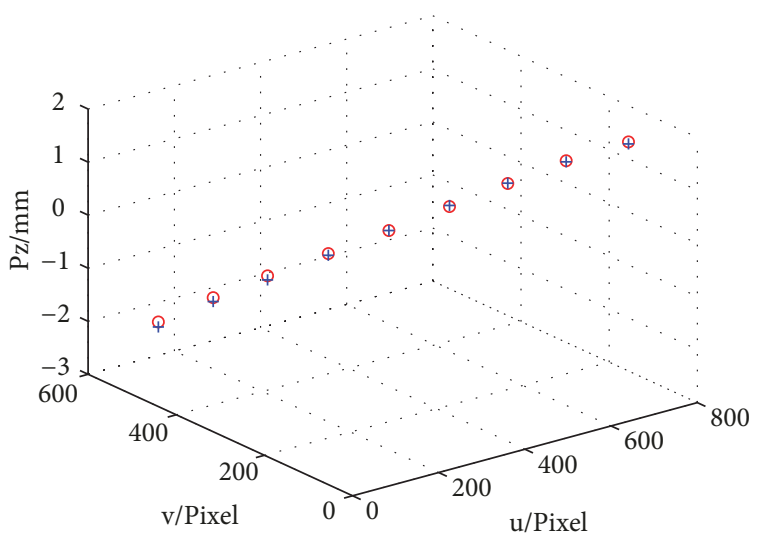

- Measured value

+ Caculated value

FIgURe 22: Pixel coordinates $u, v$ of P3 light spot and the depth $P_{z}$ of the object points under the condition of white paper surface of $0^{\circ}$.

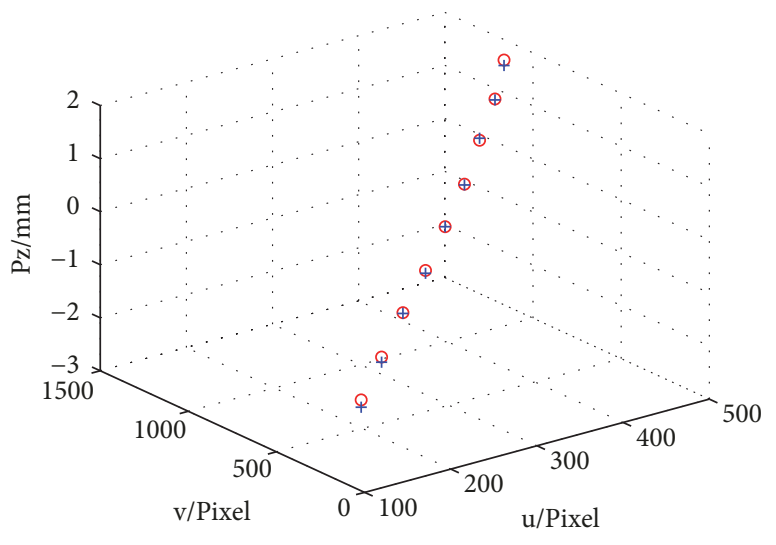

- Measured value

+ Caculated value

FIGURE 23: Pixel coordinates $u, v$ of $\mathrm{P} 4$ light spot and the depth $P_{z}$ of the object points under the condition of metal surface of $0^{\circ}$.

From the data in Figures 25 and 26, we can see that there is an error between the calculated values and the measured values. The greater the depth of the point is, the greater the measurement error of the system is. However, the error consistently fluctuate within a certain range, which is reasonable. The accuracy experiment shows that the system owns a resolution better than $0.039 \mathrm{~mm}$ under different imaging conditions.

In addition, according to the data shown in Figure 22, the linearity as well as sensitivity of the system is obtained based on the fitting straight line shown in Figure 27. Results show that the system owns good static characteristics with linearity of $0.096 \%$ and sensitivity of 1 .

4.4. Experiment of On-Machine Measurement. The probe was finally mounted on-machine. A step block which is composed of two 1-level blocks was arranged for the experiment. As

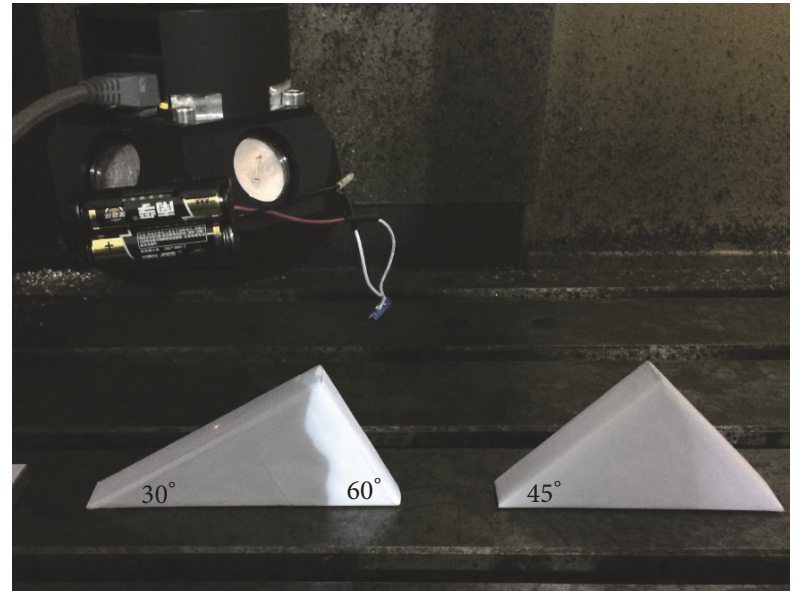

FIGURE 24: Measurement of tilted surface of $0^{\circ}, 30^{\circ}$, and $60^{\circ}$.

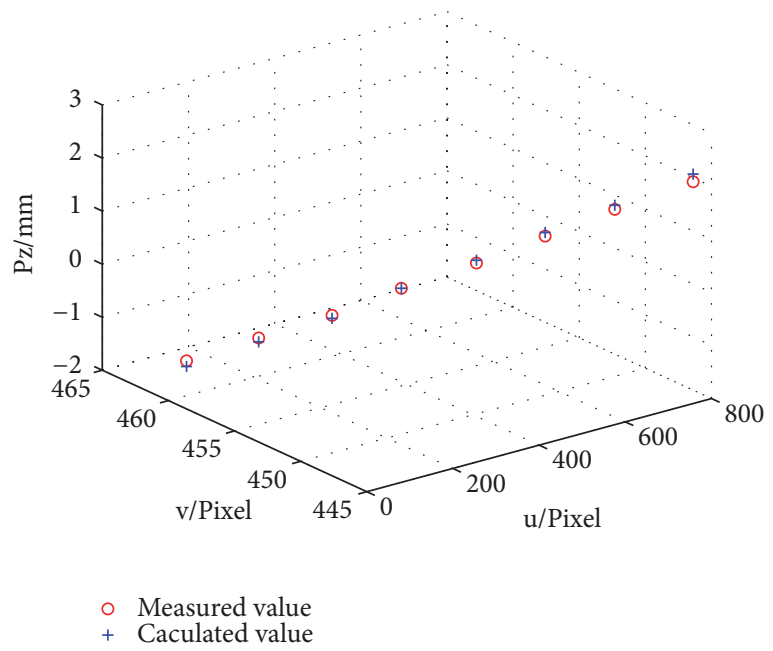

FIgURE 25: Pixel coordinates $u$, $v$ of P5 light spot and the depth $P_{z}$ of the object points under the condition of white paper surface of $30^{\circ}$.

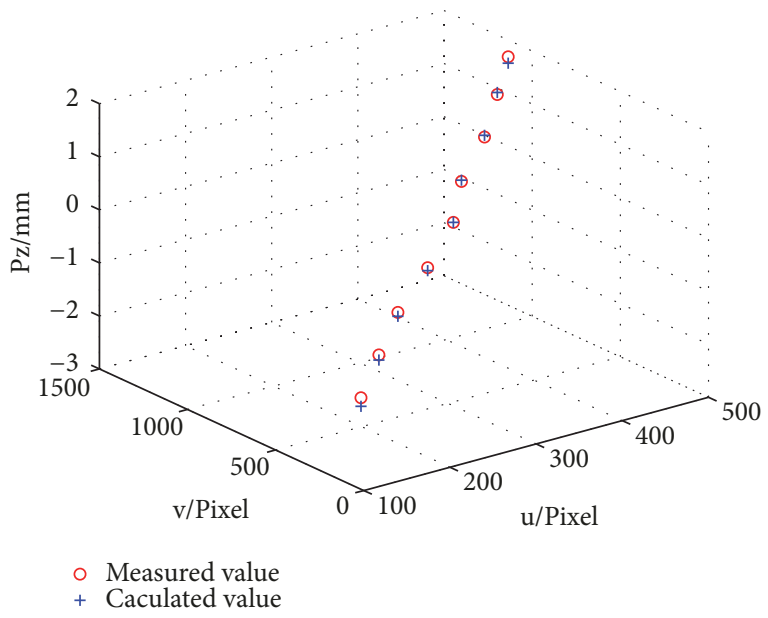

Figure 26: Pixel coordinates $u, v$ of P4 light spot and the depth $P_{z}$ of the object points under the condition of metal surface of $30^{\circ}$. 


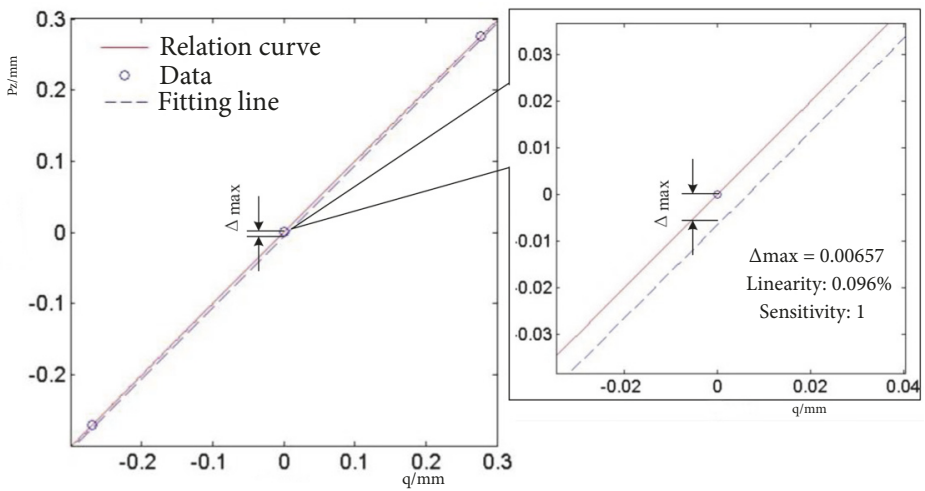

FIGURE 27: Linearity and sensitivity of the system.

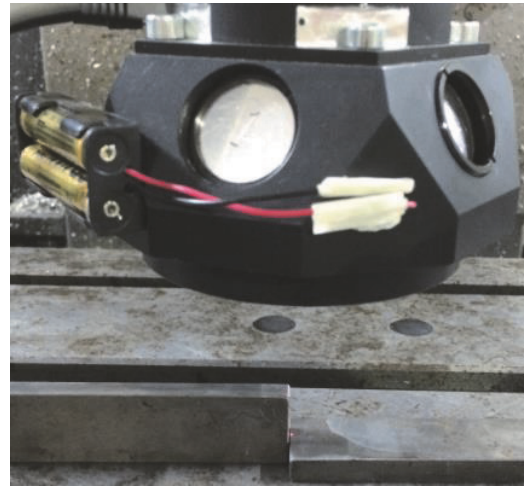

(a)

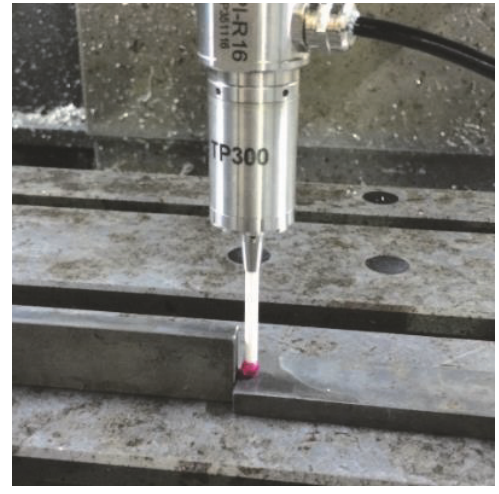

(b)

FiguRE 28: Experiment of step surface measurement.

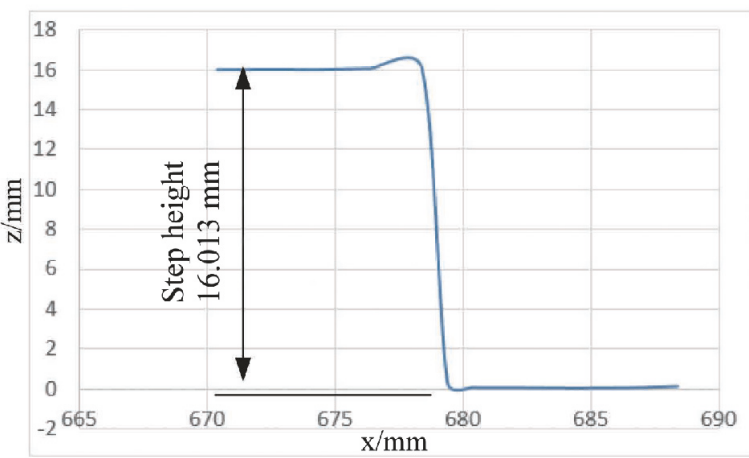

(a)

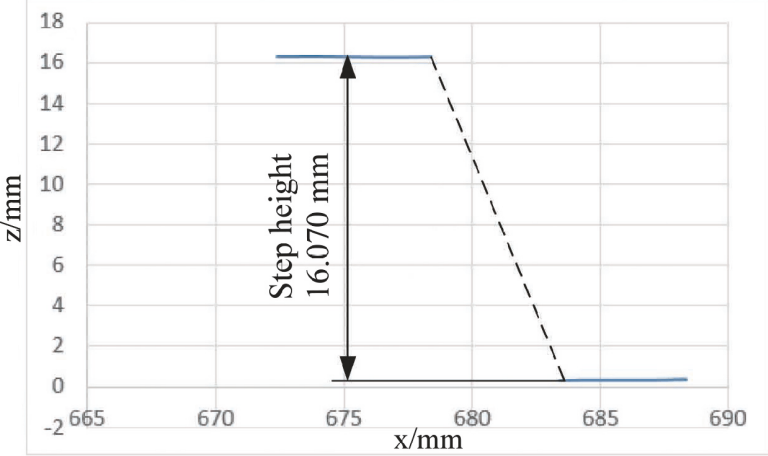

(b)

FiguRE 29: Data curve of the measurement.

is shown in Figure 28, the block with the height difference of $16 \mathrm{~mm}$ was, respectively, measured by triangulation probe and another on-machine TP300 touch probe. TP300 is a Harbin Pioneer probe used for machine tools, which owns a single track repeatability at the stylus end of $0.001 \mathrm{~mm}$. Figure 29 shows data collected by the two probes. Figure 29(a) shows data curve of the triangulation probe. There are small fluctuations at the phase position, which results from the noise produced at critical position on the step surface, but soon it restored. Figure 29(b) is the data curve of the contact probe. Due to the effect of the probe stylus, the curve has no data in the transition area between the low surface and the high surface, resulting in a blind area, as the dotted line shows in Figure 29(b). The step surface is measured as 16.013 $\mathrm{mm}$ by triangulation probe and $16.070 \mathrm{~mm}$ by TP300. The deviation of them with the theoretical value is, respectively, $0.013 \mathrm{~mm}$ and $0.070 \mathrm{~mm}$. By comparison, TP300 touch probe faces more difficulty in collecting data due to the influence 


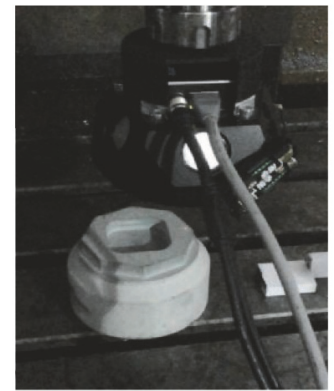

(a)

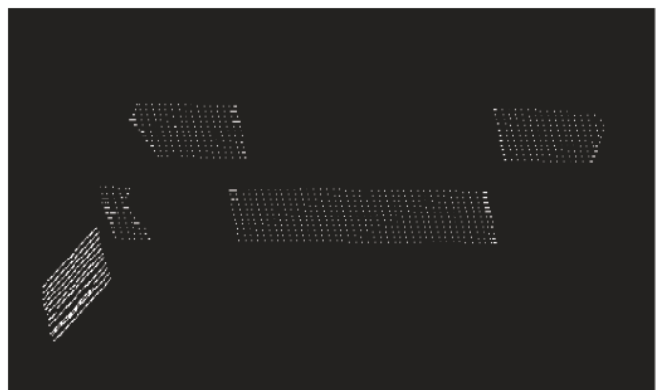

(b)

FIgURE 30: Measurement of general workpiece surface.

of its contact stylus while triangulation system has almost no measuring blind area while measuring sheltered surfaces with good robustness.

The optical probe can also measure surface of workpiece with complex structure. Figure 30(a) shows a general workpiece and Figure 30(b) shows part of the point cloud of the workpiece surface obtained by the prototype of the measurement system.

\section{Conclusion}

In this paper, an optical probe based on discrete rotational symmetric triangulation method is proposed. The model was constructed and the relationship between the image point with the real depth of laser spot was also established. After identifying required parameters of the probe system, simulations were conducted using Zemax. In the simulation of optical path, the relative distance of the object surface was, respectively, set to $-3 \mathrm{~mm}, 0 \mathrm{~mm}$, and $3 \mathrm{~mm}$. It can be seen from the simulation that light spots are distributed evenly on the receiving camera in most cases except for converging at an identical point at the position of $0 \mathrm{~mm}$. Considering that surface properties of measured object such as surface texture, inclination degree, and existence of occlusion will greatly affect measuring performance, simulations in the three aspects were then conducted. Diffuse reflection surface and metal surface were used for the simulation of surface texture. The inclination degree of $5^{\circ}$ and $60^{\circ}$ for diffuse reflection surface and that of $5^{\circ}$ and $15^{\circ}$ for metal surface were arranged for the simulation of inclination degree. Light, respectively, hitting a lower surface and hitting the critical position of a step block, was used for simulation of existence of occlusion. Results of simulations indicate that the probe can theoretically detect clear light spots in all circumstance. Even when occlusion occurs, the probe can detect at least one clear light spot for measurement.

The center-of-gravity method, which is used for the extraction of light spot center in this paper, was compared with the peak subpixel method. Tests in terms of extracting repeatability of five points and extracting stability of a certain image point both indicate the superiority of the center-ofgravity method for spot center extraction. After conducting linear calibration for the system, experiments were conducted for investigating the performance of the system, including the single point measurement for the repeatability accuracy of the system and the accuracy measurement for the resolution and static characteristics of the system. Single point measurement was divided into 8 classes according to the various combinations of surface textures and inclination degrees. The repeatability accuracy of the system was calculated about $0.72 \mu \mathrm{m}$ based on the experiment result. In the accuracy measurement, pixel coordinate of light spot and depth $P_{z}$ of the corresponding object point were shown. The system was shown to have a resolution better than $39 \mu \mathrm{m}$ and owns good static characteristics with linearity of $0.096 \%$ and sensitivity of 1 under various conditions. Finally, on-machine measurement was conducted; both the optical probe and an TP300 touch probe were used to measure a step block; the measuring result shows that the probe in this paper owns higher accuracy without blind area.

It is a universally challenging problem to measure specular surface with noncontact optical probe. To test the performance of the probe in this respect, simulations and experiments on metal surface with various inclination degrees were conducted. For the purpose of testing the performance of the probe in the case of measuring objects with occlusion effect, the simulation on a step block and the contrast on-machine experiment were conducted. Results have shown the probe has good practicability and feasibility while measuring objects with occlusion effect and specular surfaces. Due to its multidirectional imaging effect, the probe mounted on CMM (Coordinate Measuring Machine), CNC (Computer Numerical Control) machine, and other automation equipment enable measuring objects of various characteristics with good robustness.

\section{Data Availability}

The data used to support the findings of this study are available from the corresponding author upon request.

\section{Conflicts of Interest}

The authors declare that they have no conflicts of interest. 


\section{Acknowledgments}

This research was partially supported by The key research project of Ministry of Science and Technology (Grant no. 2017YFB1301503) and the National Nature Science Foundation of China (Grants no. 51575332 and no. 51605271).

\section{References}

[1] W. P. Van Vliet and P. H. J. Schellekens, "Development of a fast mechanical probe for coordinate measuring machines," Precision Engineering, vol. 22, no. 3, pp. 141-152, 1998.

[2] W.-L. Li, G. Wang, G. Zhang, Q.-D. Li, and Z.-P. Yin, "Interference-free inspection path generation for impeller blades using an on-machine probe," IEEE/ASME Transactions on Mechatronics, vol. 22, no. 3, pp. 1218-1226, 2017.

[3] M. Del Guerra and R. T. Coelho, "Development of a low cost Touch Trigger Probe for CNC Lathes," Journal of Materials Processing Technology, vol. 179, no. 1-3, pp. 117-123, 2006.

[4] T. Oiwa and T. Tanaka, "Miniaturized three-dimensional touch trigger probe using optical fibre bundle," Measurement Science and Technology, vol. 16, no. 8, pp. 1574-1581, 2005.

[5] A. Weckenmann and J. Hoffmann, Probing Systems for Coordinate Measuring Machines, 2011.

[6] http://www.marposs.com/technology.php/eng/touch_probes_ machine_tools.

[7] R.-J. Li, K.-C. Fan, Q.-X. Huang, H. Zhou, E.-M. Gong, and M. Xiang, "A long-stroke 3D contact scanning probe for micro/nano coordinate measuring machine," Precision Engineering, vol. 43, pp. 220-229, 2016.

[8] F. Meli and A. Kng, "Afm investigation on surface damage caused by mechanical probing with small ruby spheres," Measurement Science \& Technology, vol. 18, no. 18, p. 496, 2007.

[9] C. Butler, "An investigation into theperformance of probes on coordinate measuring machines," Industrial Metrology, vol. 2, no. 1, pp. 59-70, 1991.

[10] S. Srinivasan, Y. Kovvur, and S. Anand, "Probe radius compensation for On-Machine Measurement of sculptured surfaces," in Proceedings of the 2004 ASME International Mechanical Engineering Congress and Exposition, IMECE 2004, pp. 913-920, November 2004.

[11] R. T. Lee and F. J. Shiou, "Calculation of the unit normal vector using the cross-curve moving mask method for probe radius compensation of a freeform surface measurement," Measurement, vol. 43, no. 4, pp. 469-478, 2010.

[12] M. Ristic, I. Ainsworth, and D. Brujic, "Contact probe radius compensation using computer aided design models," Proceedings of the Institution of Mechanical Engineers, Part B: Journal of Engineering Manufacture, vol. 215, no. 6, pp. 819-834, 2001.

[13] J. R. R. Mayer, Y. A. Mir, F. Trochu, A. Vafaeesefat, and M. Balazinski, "Touch probe radius compensation for coordinate measurement using kriging interpolation," Proceedings of the Institution of Mechanical Engineers, Part B: Journal of Engineering Manufacture, vol. 211, no. 1, pp. 11-18, 1997.

[14] M. Silvestri, M. Banfi, A. Bettoni, M. Confalonieri, A. Ferrario, and M. Floris, Use of Laser Scanners in Machine Tools to Implement Freeform Parts Machining and Quality Control, Springer International Publishing, 2016.

[15] X. Zou, X. Zhao, G. Li, Z. Li, and T. Sun, "Non-contact onmachine measurement using a chromatic confocal probe for an ultra-precision turning machine," The International Journal of
Advanced Manufacturing Technology, vol. 90, no. 5-8, pp. 21632172, 2016.

[16] L. A. Cacace, An Optical Distance Sensor:Tilt Robust Differential Confocal Measurement with $\mathrm{mm}$ Range and nm Uncertainty, Technische Universiteit Eindhoven, 2009.

[17] A. Harasaki and J. C. Wyant, "Fringe modulation skewing effect in white-light vertical scanning interferometry," Applied Optics, vol. 39, no. 13, pp. 2101-2106, 2000.

[18] F. Gao, R. K. Leach, J. Petzing, and J. M. Coupland, "Surface measurement errors using commercial scanning white light interferometers," Measurement Science and Technology, vol. 19, no. $1,2008$.

[19] P. Ott, "Imaging with tilted surfaces: an efficient matrix method for the generalized scheimpflug condition and its application to rotationally symmetric triangulation," Journal of the Optical Society of America A Optics Image Science \& Vision, vol. 22, no. 6, p. 1077, 2005.

[20] M. Kujawinska, "Optical design of rotationally symmetric triangulation sensors with low-cost detectors based on reflective optics," in Proceedings of the SPIE - The International Society for Optical Engineering, vol. 5144, pp. 350-359, 2003.

[21] J. Eckstein, J. Gao, P. Ott, W. Lei, and X. Wang, "New compact rotationally symmetric triangulation sensor with low-cost plastic optics," International Congress on Optics and Optoelectronics, 2007.

[22] W. Tao, "Rotational symmetric triangulation sensor based on an object space mirror," Optical Engineering, vol. 50, no. 12, p. 4401, 2011.

[23] A. Cigada, F. Mancosu, S. Manzoni, and E. Zappa, "Lasertriangulation device for in-line measurement of road texture at medium and high speed," Mechanical Systems and Signal Processing, vol. 24, no. 7, pp. 2225-2234, 2010.

[24] S. Ibaraki and Y. Nagai, "Formulation of the influence of rotary axis geometric errors on five-axis on-machine optical scanning measurement-application to geometric error calibration by "chase-the-ball" test," The International Journal of Advanced Manufacturing Technology, vol. 92, no. 9-12, pp. 4263-4273, 2017.

[25] M. Demeyere, D. Rurimunzu, and C. Eugène, "Diameter measurement of spherical objects by laser triangulation in an ambulatory context," IEEE Transactions on Instrumentation and Measurement, vol. 56, no. 3, pp. 867-872, 2007.

[26] M. Ishihara and H. Sasaki, "High-speed surface measurement using a nonscanning multiple-beam confocal microscope," Optical Engineering, vol. 38, no. 6, pp. 1035-1040, 1999.

[27] M. Munaro, S. Michieletto, E. So, D. Alberton, and E. Menegatti, "Fast $2.5 \mathrm{~d}$ model reconstruction of assembled parts with high occlusion for completeness inspection," in Proceedings of the International Conference on Machine Vision, Image Processing, and Pattern Analysis, 2011.

[28] E. W. Y. So, M. Munaro, S. Michieletto, M. Antonello, and E. Menegatti, "Real-time 3d model reconstruction with a dual-laser triangulation system for assembly line completeness inspection," in Proceedings of the Intelligent Autonomous Systems Conference, 2012.

[29] L. Zeng, H. Matsumoto, and K. Kawachi, "Two-directional scanning method for reducing the shadow effects in laser triangulation," Measurement Science and Technology, vol. 8, no. 3, pp. 262-266, 1997.

[30] K. N. Kutulakos and E. Steger, "A theory of refractive and specular 3D shape by light-path triangulation," in Proceedings 
of the Proceedings - 10th IEEE International Conference on Computer Vision, ICCV 2005, vol. 2, pp. 1448-1455, October 2005.

[31] T. Bonfort, P. Sturm, and P. Gargallo, "General specular surface triangulation," Lecture Notes in Computer Science (including subseries Lecture Notes in Artificial Intelligence and Lecture Notes in Bioinformatics): Preface, vol. 3852, pp. 872-881, 2006. 


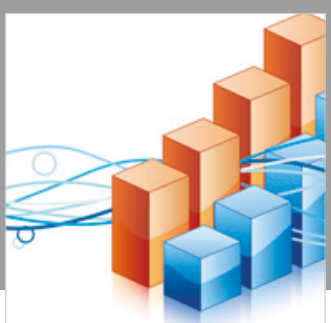

Advances in

Operations Research

\section{-n-m}
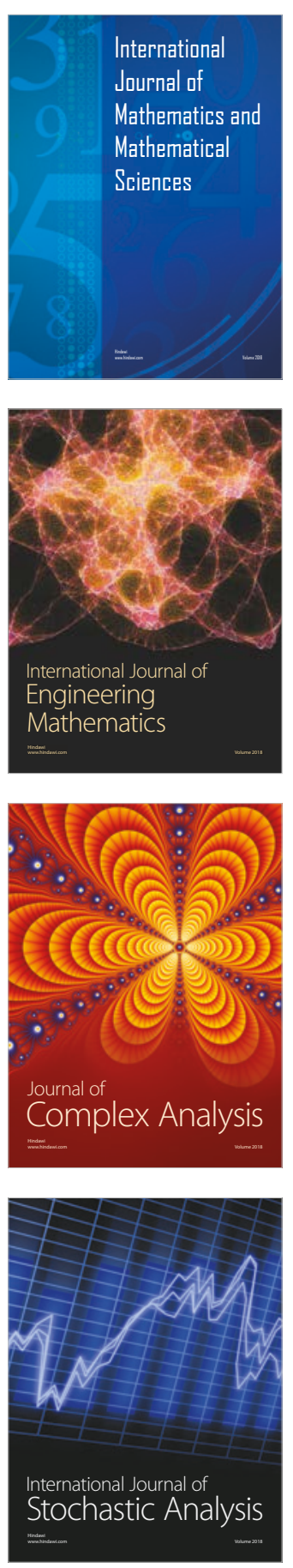
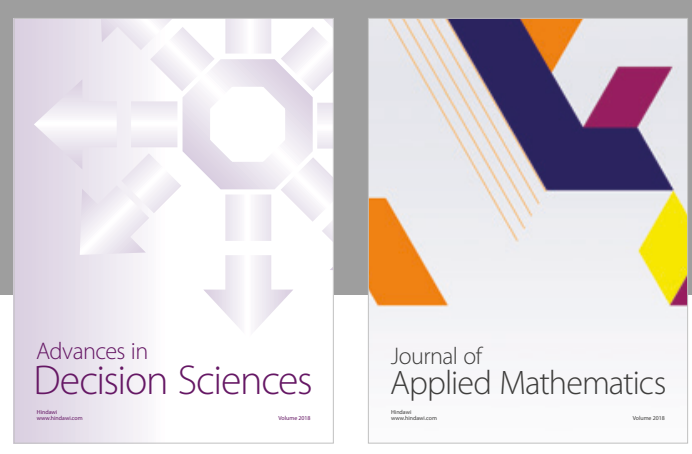

Journal of

Applied Mathematics
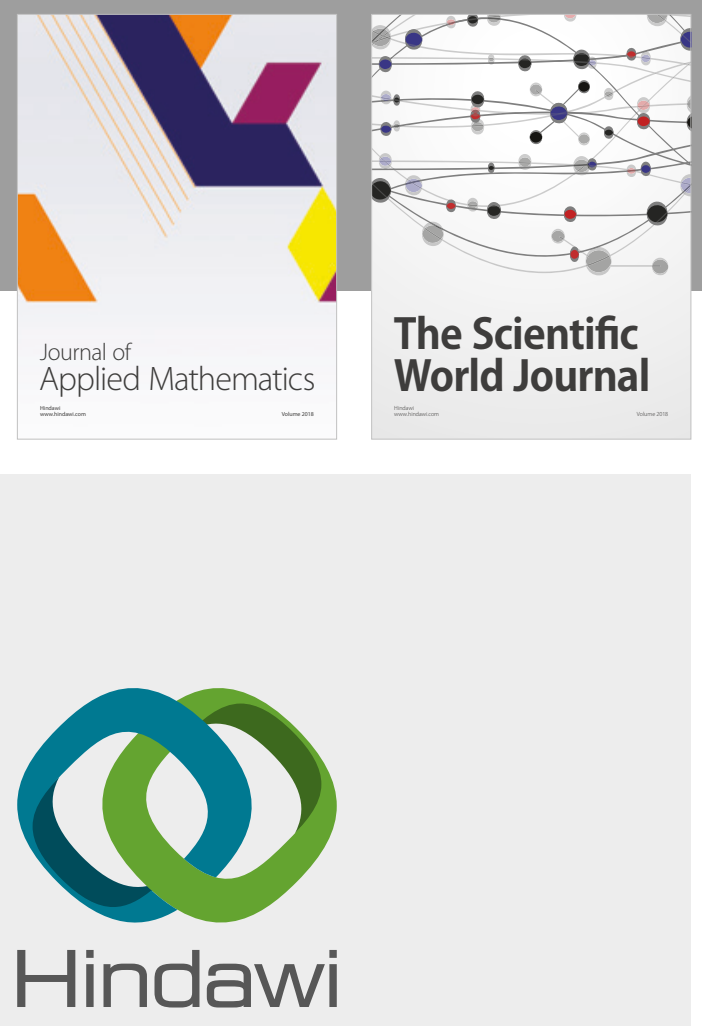

Submit your manuscripts at

www.hindawi.com

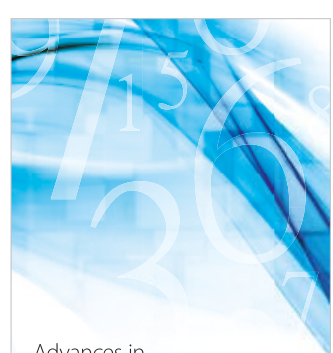

Advances in
Numerical Analysis
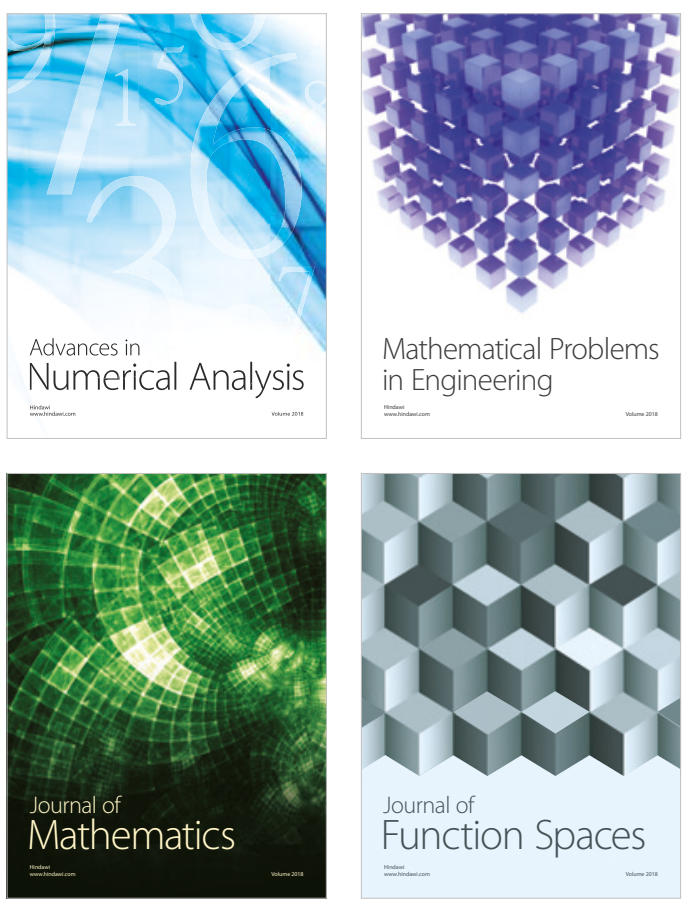

Mathematical Problems in Engineering

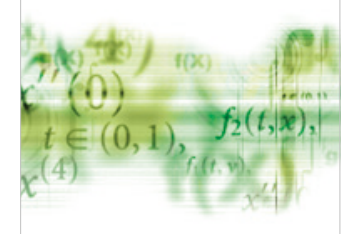

International Journal of

Differential Equations

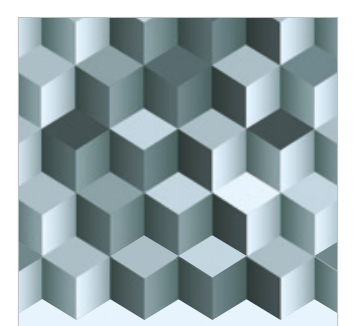

Journal of

Function Spaces

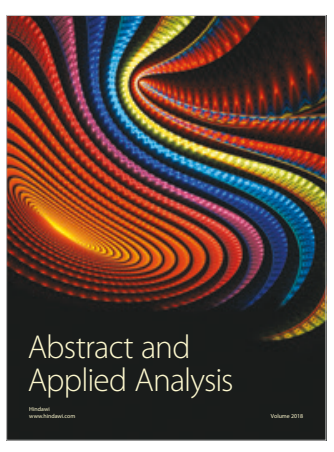

The Scientific

World Journal

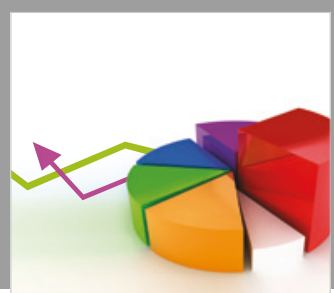

Journal of

Probability and Statistics
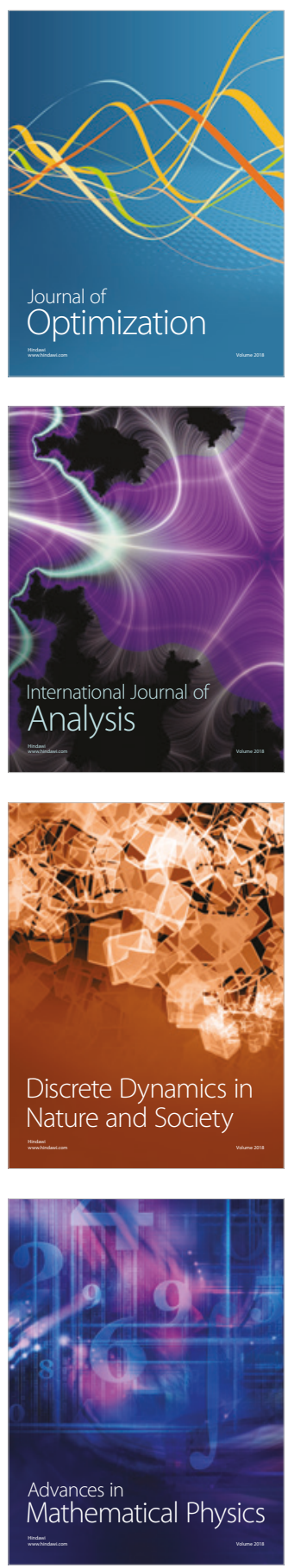